\title{
Radio spectra of bright compact sources at $\mathrm{z}>4.5$
}

\author{
Rocco Coppejans ${ }^{1 \star}$, Sjoert van Velzen ${ }^{2}$, Huib T. Intema ${ }^{3}$, Cornelia Müller ${ }^{1}$, \\ Sándor Frey ${ }^{4,8}$, Deanne L. Coppejans ${ }^{1}$, Dávid Cseh $^{1}$, Wendy L. Williams ${ }^{5}$, \\ Heino Falcke ${ }^{1,6}$, Elmar G. Körding ${ }^{1}$, Emanuela Orrú ${ }^{6,1}$, Zsolt Paragi ${ }^{7}$, \\ and Krisztina É. Gabányi ${ }^{4,8}$ \\ ${ }^{1}$ Department of Astrophysics/IMAPP, Radboud University, P.O. Box 9010, 6500 GL Nijmegen, The Netherlands \\ ${ }^{2}$ Department of Physics and Astronomy, The Johns Hopkins University, Baltimore, MD 21218, USA \\ ${ }^{3}$ Leiden Observatory, Leiden University, PO Box 9513, 2300 RA, Leiden, The Netherlands \\ ${ }^{4}$ FÖMI Satellite Geodetic Observatory, PO Box 585, H-1592 Budapest, Hungary \\ ${ }^{5}$ School of Physics, Astronomy and Mathematics, University of Hertfordshire, College Lane, Hatfield AL10 $9 A B$, UK \\ ${ }^{6}$ Netherlands Institute for Radio Astronomy (ASTRON), PO Box 2, 7990 AA Dwingeloo, The Netherlands \\ ${ }^{7}$ Joint Institute for VLBI ERIC, Postbus 2, 7990 AA Dwingeloo, The Netherlands \\ ${ }^{8}$ Konkoly Observatory, MTA Research Centre for Astronomy and Earth Sciences, Konkoly Thege Miklós út 15-17, H-1121 Budapest, Hungary
}

\begin{abstract}
High-redshift quasars are important to study galaxy and active galactic nuclei (AGN) evolution, test cosmological models, and study supermassive black hole growth. Optical searches for high-redshift sources have been very successful, but radio searches are not hampered by dust obscuration and should be more effective at finding sources at even higher redshifts. Identifying high-redshift sources based on radio data is, however, not trivial. Here we report on new multi-frequency Giant Metrewave Radio Telescope (GMRT) observations of eight $z>4.5$ sources previously studied at high angular resolution with very long baseline interferometry (VLBI). Combining these observations with those from the literature, we construct broad-band radio spectra of all $30 z>4.5$ sources that have been observed with VLBI. In the sample we found flat, steep and peaked spectra in approximately equal proportions. Despite several selection effects, we conclude that the $z>4.5$ VLBI (and likely also non-VLBI) sources have diverse spectra and that only about a quarter of the sources in the sample have flat spectra. Previously, the majority of high-redshift radio sources were identified based on their ultra-steep spectra (USS). Recently a new method has been proposed to identify these objects based on their megahertz-peaked spectra (MPS). Neither method would have identified more than 18 per cent of the high-redshift sources in this sample. More effective methods are necessary to reliably identify complete samples of high-redshift sources based on radio data.
\end{abstract}

Key words: radio continuum: galaxies - galaxies: active - galaxies: high-redshift

\section{INTRODUCTION}

It is believed that there is a supermassive black hole at the center of nearly every galaxy. These objects power active galactic nuclei (AGN) and were formed in the early Universe. They continue to influence, shape, and grow with their host galaxy via feedback (e.g. Best et al. 2005; Fabian 2012; Morganti et al. 2013). To understand present-day galaxies, we consequently need to understand AGN evolution (e.g.

\footnotetext{
* E-mail: r.coppejans@astro.ru.nl
}

Fabian 2012). A critical aspect of this is identifying AGN at high redshifts.

In the optical, AGN have been found at distances of up to redshift 7.1 (Mortlock et al. 2011). However, due to Lyalpha absorption, detecting sources beyond $z=6.5$ is very difficult in the optical (Mortlock et al. 2011; Becker et al. 2001). In addition optical searches are hampered by dust obscuration, which does not affect radio observations (e.g. Osmer 2004). With radio observations, we should therefore be able to detect sources at all redshifts more effectively, and detect sources out to higher redshifts. It is worth noting that 
optical spectroscopy is still essential to determine redshifts of the candidate high-redshift sources detected in the radio.

One of the main techniques that is used to identify highredshift sources in radio images, is the ultra-steep-spectrum (USS) method. This method is based on an observed correlation between the spectral index ( $\alpha$; defined as $S \propto \nu^{\alpha}$ where $S$ is the flux density at frequency $\nu$ ) and redshift (e.g. Whitfield 1957; Blumenthal \& Miley 1979; Laing \& Peacock 1980; De Breuck et al. 2000). According to this correlation, sources that have steeper spectra are at higher redshifts. The USS method has proven successful: most of the high-redshift sources identified through radio observations were selected using this method (De Breuck et al. 2000; Verkhodanov \& Khabibullina 2010; Singh et al. 2014), and it has also succeeded in finding sources out to $z>4$ (e.g. Van Breugel et al. 1999; Jarvis et al. 2001; Kopylov et al. 2006).

Despite this success, there is no physical explanation for why USS sources should be at higher redshifts than nonUSS sources (e.g. Miley \& De Breuck 2008; Klamer et al. 2006; Verkhodanov \& Khabibullina 2010; Singh et al. 2014), and several recent studies have failed to find a correlation between the spectral index and redshift (Ker et al. 2012; Singh et al. 2014; Smolčić et al. 2014). The exact definition of a USS source (based on spectral index) differs between authors, e.g., $\alpha_{327 \mathrm{MHz}}^{608 \mathrm{MHz}}<-1.1$ (Wieringa \& Katgert 1992), $\alpha_{151 \mathrm{MHz}}^{4.85 \mathrm{GHz}}<-0.981$ (Blundell et al. 1998), $\alpha_{843 \mathrm{MHz}}^{1.4 \mathrm{GHz}}<-1.3$ (De Breuck et al. 2004), $\alpha_{151 \mathrm{MHz}}^{1.4 \mathrm{GHz}}<-1.0$ (Cruz et al. 2006), $\alpha_{408 \mathrm{MHz}}^{843 \mathrm{MHz}} \leqslant-1.0$ (Broderick et al. 2007) and $\alpha_{325 \mathrm{MHz}}^{1.4 \mathrm{GHz}} \leqslant-1.0$ (Singh et al. 2014). However, Coppejans et al. (2015) pointed out that in their sample of sources, in which all of the sources are detected at 153,325 and $1400 \mathrm{MHz}$, when first selecting USS sources between 153 and $325 \mathrm{MHz}$ and then selecting USS sources between 325 and $1400 \mathrm{MHz}$, less than 26 per cent of the sources appear in both selections. Pedani (2003) has also pointed out that the USS sources may not be representative of the entire high-redshift source population, since USS sources are typically smaller and more powerful than non-USS sources (Blundell et al. 1999). This argument is supported by the discovery of two non-USS sources at $z=4.4$ and 4.9 with $\alpha_{1.4 \mathrm{GHz}}^{8.5 \mathrm{GHz}}=0.94 \pm 0.06$ and $\alpha_{325 \mathrm{MHz}}^{1.4 \mathrm{GHz}}=0.75 \pm 0.05$, respectively, (Waddington et al. 1999; Jarvis et al. 2009). Pedani (2003) has shown that up to 40 per cent of the high-redshift sources in a survey can be lost by applying a spectral index cut.

Falcke et al. (2004) and Coppejans et al. (2015) proposed a new method for searching for high-redshift AGN, namely the megahertz peaked-spectrum (MPS) method. Compact steep-spectrum (CSS), MPS, gigahertz peakedspectrum (GPS) and high-frequency peaked (HFP) sources are all AGN that show spectral turnovers in their synchrotron spectra, that are believed to be produced by synchrotron self-absorption. GPS, MPS and CSS sources together make up between 15 and 30 per cent of the sources in flux density limited catalogues (O'Dea 1998; Orienti 2016). The observed turnover (or peak) frequencies $\left(\nu_{\mathrm{o}}\right)$ of the CSS, MPS, GPS and HFP sources are $\nu_{\mathrm{o}}<0.5 \mathrm{GHz}, \nu_{\mathrm{o}}<1 \mathrm{GHz}$, $1<\nu_{\mathrm{o}}<5 \mathrm{GHz}$ and $\nu_{\mathrm{o}}>5 \mathrm{GHz}$ (O'Dea 1998; Dallacasa et al. 2000; Coppejans et al. 2015), respectively. These sources are believed to be young (rather than confined) AGN, some of which will likely evolve into FR I and FR II radio galaxies (Begelman 1996; O'Dea 1998; Snellen et al. 2000; Conway 2002; De Vries et al. 2002; Murgia et al. 2002;
Murgia 2003; Fanti 2009; An \& Baan 2012; Orienti 2016). For the nearby $(z \sim 1)$ CSS, MPS, GPS and HFP sources, an empirical relation exists between the rest-frame turnover frequencies $\left(\nu_{\mathrm{r}}\right.$, where $\left.\nu_{\mathrm{r}}=\nu_{\mathrm{o}}(1+z)\right)$ and the linear sizes of the sources (O'Dea 1998; Snellen et al. 2000; Orienti \& Dallacasa 2014). From this relation, sources with lower values of $\nu_{\mathrm{r}}$ have larger linear sizes.

The premise of the MPS method is that there are two classes of sources that have peak frequencies below $1 \mathrm{GHz}$. The first class, which includes the CSS sources, are nearby sources for which $\nu_{\mathrm{o}} \simeq \nu_{\mathrm{r}}$. The second class of sources have $\nu_{\mathrm{r}}>1 \mathrm{GHz}$, but $\nu_{\mathrm{o}}<1 \mathrm{GHz}$ due to their higher redshifts. There are two differences between these two classes. First, we expect the high-redshift sources to have smaller angular sizes than the CSS sources, as they are at larger redshifts. Second, the high-redshift sources have higher restframe turnover frequencies than the nearby sources. From the turnover frequency-linear size relation, we therefore expect the high-redshift sources to have smaller physical sizes than the CSS sources. It should therefore be possible to distinguish between the CSS and the high-redshift sources based on the high-redshift sources having smaller angular sizes than the CSS sources.

To date no new high-redshift sources have been found using the MPS method. However, Coppejans et al. (2015) identified 33 MPS sources in the NOAO Boötes field and were able to determine redshifts for 24 . Given that the average redshift of the sources is 1.3 , that there are five sources at $z>2$ and that four of the sources for which they could not find redshifts are likely also at $z>2$, the authors concluded that there is encouraging evidence in support of the method. Like the USS method, the MPS method likely only selects a subset of the high-redshift sources. However, the MPS method selects a different class of high-redshift sources than the USS method as it is believed that the MPS sources are young AGN (O'Dea 1998; Murgia et al. 2002; Conway 2002). For this reason, the MPS method is important for understanding AGN evolution. The two methods are therefore complementary and will allow for a better understanding of the high-redshift population as a whole.

In Coppejans et al. (2016, hereafter CFC2016), we presented very long baseline interferometry (VLBI) observations of ten new $z>4.5$ sources at 1.7 and $5 \mathrm{GHz}$ with the European VLBI Network (EVN). This increased the number of $z>4.5$ sources that have been observed with VLBI by 50 per cent, from 20 to 30 sources. Using both the VLBI brightness temperatures and $1.4 \mathrm{GHz}$ luminosities of all $30 z>4.5$ VLBI sources, we concluded that in one of the sources the radio emission is from star formation, with the emission originating from AGN activity in the other 29 sources $^{1}$. This illustrates that even at $z>4.5$, not all sources detected with VLBI are AGN. From the VLBI spec-

1 Typically, brightness temperatures $\left(T_{\mathrm{b}}\right)$ above $10^{6} \mathrm{~K}$ indicate non-thermal emission from AGN (e.g. Kewley et al. 2000; Middelberg et al. 2011) while thermal emission from star formation has $T_{\mathrm{b}}<10^{5} \mathrm{~K}$ (Sramek \& Weedman 1986; Condon et al. 1991; Kewley et al. 2000). In Magliocchetti et al. (2014) the authors showed that at $z>1.8$ the radio emission in sources with $1.4 \mathrm{GHz}$ radio luminosities above $4 \times 10^{24} \mathrm{~W} \mathrm{~Hz}^{-1}$ is caused by AGN activity, while the radio emission in sources with $1.4 \mathrm{GHz}$ radio luminosities lower than $4 \times 10^{24} \mathrm{~W} \mathrm{~Hz}^{-1}$ is caused by star formation. 
tra, brightness temperatures, and $1.4 \mathrm{GHz}$ variability we also concluded that the $z>4.5$ VLBI sources are a mixture of steep-spectrum sources and flat-spectrum radio quasars (FSRQs), or blazars, i.e. sources in which the jet is aligned within a small angle of our line of sight (e.g. Urry 1999; Krawczynski \& Treister 2013). We finally argued that the steep-spectrum sources are in fact GPS and MPS sources.

In this paper, we continue our study of all $30 z>4.5$ VLBI sources by investigating their broad-band radio spectra. The sources were collected from the Optical Characteristics of Astrometric Radio Sources (OCARS) cata$\operatorname{logue}^{2}$ (Malkin \& Titov 2008; Malkin 2016) and the literature. To the best of our knowledge, these 30 sources are the only sources with spectroscopic redshifts above 4.5 that have been imaged with VLBI. We restricted ourselves to only studying sources that have been observed with VLBI in this paper for the following reasons: (1) VLBI observations are necessary to get accurate brightness temperatures for the sources. As discussed in CFC2016, this allows us to distinguish between emission from AGN and star formation and is critical to explain the spectra of J1429+5447 and J1205-0742 in Sections 4.2.6 and 4.4.2. (2) The $z>4.5$ VLBI sources can be seen as forming a flux density limited sample since all $z>4.5$ sources with $1.4 \mathrm{GHz}$ flux densities above $\sim 5 \mathrm{mJy}$ in the Very Large Array (VLA) Faint Images of the Radio Sky at Twenty-centimeter (FIRST) survey (White et al. 1997) have been systematically observed with VLBI in published (Coppejans et al. 2016, and references therein) and ongoing VLBI campaigns. We do however note that some authors have specifically targeted fainter sources. In addition not all $z>4.5$ sources with FIRST flux densities above $5 \mathrm{mJy}$ are included in our sample of sources, as these sources were only identified as $z>4.5$ sources after the EVN observing proposal for Coppejans et al. (2016) had been submitted. These sources are currently being observed in our latest series of EVN observations. (3) This paper is a continuation of the work in CFC2016. The redshifts and VLBI positions of all of the sources are given in Table 1. The VLBI positions are taken from the highest frequency VLBI observations (listed in Table 5) of the sources as these observations will have the highest positional accuracy.

For a source at $z=4.5$, its entire rest-frame spectrum below $5.5 \mathrm{GHz}$ will be redshifted into observed frequencies below $1 \mathrm{GHz}$. Consequently, to accurately characterize the spectrum, multi-frequency observations of the source below $1 \mathrm{GHz}$ are required. In Section 2, we present multi-frequency Giant Metrewave Radio Telescope (GMRT) observations below $1 \mathrm{GHz}$ of eight $z>4.5$ sources that have been observed at two frequencies with the EVN. Section 3 contains a description of how we matched all $30 z>4.5 \mathrm{VLBI}$ sources to previous radio observations. The spectra and classifications are presented for each source individually in Section 4. In Section 5 we discuss the spectral classification of the $z>4.5$ VLBI sources, before presenting a summary and conclusion in Section 6. Throughout this paper we assume the following cosmological model parameters: $\Omega_{\mathrm{m}}=0.3, \Omega_{\lambda}=0.7$, $H_{0}=72 \mathrm{~km} \mathrm{~s}^{-1} \mathrm{Mpc}^{-1}$.

2 http://www.gao.spb.ru/english/as/ac_vlbi/ocars.txt
Table 1. Source redshifts and positions

\begin{tabular}{cccc}
\hline ID & $z$ & RA [J2000] & DEC [J2000] \\
\hline J0011+1446 & 4.96 & $00: 11: 15.233$ & $14: 46: 01.81$ \\
J0131-0321 & 5.18 & $01: 31: 27.347$ & $-03: 21: 00.08$ \\
J0210-0018 & 4.65 & $02: 10: 43.164$ & $-00: 18: 18.44$ \\
J0311+0507 & 4.51 & $03: 11: 47.966$ & $05: 08: 03.87$ \\
J0324-2918 & 4.63 & $03: 24: 44.295$ & $-29: 18: 21.22$ \\
J0813+3508 & 4.92 & $08: 13: 33.327$ & $35: 08: 10.77$ \\
J0836+0054 & 5.77 & $08: 36: 43.860$ & $00: 54: 53.23$ \\
J0906+6930 & 5.47 & $09: 06: 30.750$ & $69: 30: 30.80$ \\
J0913+5919 & 5.11 & $09: 13: 16.547$ & $59: 19: 21.67$ \\
J0940+0526 & 4.50 & $09: 40: 04.800$ & $05: 26: 30.95$ \\
J1013+2811 & 4.75 & $10: 13: 35.440$ & $28: 11: 19.24$ \\
J1026+2542 & 5.27 & $10: 26: 23.621$ & $25: 42: 59.43$ \\
J1146+4037 & 5.01 & $11: 46: 57.790$ & $40: 37: 08.63$ \\
J1205-0742 & 4.69 & $12: 05: 22.977$ & $-07: 42: 29.75$ \\
J1235-0003 & 4.69 & $12: 35: 03.046$ & $-00: 03: 31.76$ \\
J1242+5422 & 4.73 & $12: 42: 30.589$ & $54: 22: 57.45$ \\
J1311+2227 & 4.61 & $13: 11: 21.321$ & $22: 27: 38.63$ \\
J1400+3149 & 4.64 & $14: 00: 25.416$ & $31: 49: 10.68$ \\
J1427+3312 & 6.12 & $14: 27: 38.585$ & $33: 12: 41.93$ \\
J1429+5447 & 6.21 & $14: 29: 52.176$ & $54: 47: 17.63$ \\
J1430+4204 & 4.72 & $14: 30: 23.742$ & $42: 04: 36.49$ \\
J1454+1109 & 4.93 & $14: 54: 59.305$ & $11: 09: 27.89$ \\
J1548+3335 & 4.68 & $15: 48: 24.014$ & $33: 35: 00.09$ \\
J1606+3124 & 4.56 & $16: 06: 08.518$ & $31: 24: 46.46$ \\
J1611+0844 & 4.54 & $16: 11: 05.650$ & $08: 44: 35.48$ \\
J1628+1154 & 4.47 & $16: 28: 30.465$ & $11: 54: 03.47$ \\
J1659+2101 & 4.78 & $16: 59: 13.228$ & $21: 01: 15.81$ \\
J1720+3104 & 4.62 & $17: 20: 26.688$ & $31: 04: 31.65$ \\
J2102+6015 & 4.58 & $21: 02: 40.219$ & $60: 15: 09.84$ \\
J2228+0110 & 5.95 & $22: 28: 43.526$ & $01: 10: 31.91$ \\
\hline Note & & & \\
\hline
\end{tabular}

Notes: a Parijskij et al. (2014) found that J0311+0507 is composed of eight components and conclude that the third component is the core. The RA and DEC values are therefore for the third component.

\section{OBSERVATIONS WITH THE GMRT}

The sources presented in Table 2 were observed with the GMRT during two projects: 21_013 and 29_007. During project 21_013 the following three sources were observed: $\mathrm{J} 1146+4037$, J1242+5422 and J1659+2101. The remaining five sources were observed during project 29_007. The sources for project 21_013 were selected from Frey et al. (2010), while the sources for project 29_007 were selected from CFC2016. In these two publications, the observations of $15 z>4.5$ sources with the EVN at 1.6 and $5 \mathrm{GHz}$, or 1.7 GHz and $5 \mathrm{GHz}$ are described. In project 21_013, sources were only considered for observation if they had steep radio spectra $(\alpha<-0.5)$ based on their VLBI flux densities. To ensure that the sources were sufficiently bright to be detected with the GMRT, in project 29_007, we selected sources based on their $1.4 \mathrm{GHz}$ flux densities in FIRST, and based on whether they were detected at 325 or $148 \mathrm{MHz}$ with the Westerbork Northern Sky Survey (WENSS; Rengelink et al. 1997) and the Tata Institute of Fundamental Research GMRT Sky Survey alternative data release 1 (TGSS; Intema et al. 2016), respectively.

During project 21_013, the observations of J1146+4037, $\mathrm{J} 1242+5422$ and $\mathrm{J} 1659+2101$ were carried out using $32 \mathrm{MHz}$ of bandwidth in the $325 \mathrm{MHz}$ band and $16 \mathrm{MHz}$ of bandwidth in the 610,235 and $150 \mathrm{MHz}$ bands. The central 
frequencies in each of these bands were 612, 322, 235 and $148 \mathrm{MHz}$. In project 29_007, J0210-0018, J0940+0526, $\mathrm{J} 1400+3149, \mathrm{~J} 1548+3335$ and $\mathrm{J} 1628+1154$ were observed using $32 \mathrm{MHz}$ of bandwidth in the 610,325 and $150 \mathrm{MHz}$ bands, which had central frequencies of 608, 323 and $148 \mathrm{MHz}$. In both projects the observations of the target sources were flanked (where possible), or preceded or followed (where not possible), by 5-10 minute observations of one or two of the following calibrator sources: 3C48, 3C147, 3C286, J1146+399, J1219+484, J1427+3312, J1506+375 and J1719+177. In total, 24.5 hours of observations were taken for project 21_013 and 13.5 hours for project 29_007.

The data were reduced using the SPAM pipeline as described by Intema et al. (2016). The flux density scale was set by $3 \mathrm{C} 48,3 \mathrm{C} 147$ or $3 \mathrm{C} 286$ and was tied to the Scaife $\&$ Heald (2012) standard with an accuracy of $\sim 10$ per cent (e.g. Chandra et al. 2004). The initial phase calibration of the target fields was done using a source model derived from the TGSS survey (Intema et al. 2016). The source parameters in Table 2 were extracted from the images using the PYBDSM source detection package (Mohan \& Rafferty 2015). As the VLBI positions of all of the sources are known (Coppejans et al. 2016, and references therein), we set the source detection threshold, defined as the source's peak brightness divided by the local root mean square (rms) noise $\left(\sigma_{\text {local }}\right)$, to $3 \sigma_{\text {local }}$. All of the sources, except J0210-0018, were detected in all the observations as single components. J0210-0018 had two components in the GMRT610 image and one component in the GMRT325 and GMRT150 images. This is discussed in detail in Section 4.1.3. Following Intema et al. (2016), the uncertainties on the flux densities in Table 2 were increased by adding 10 per cent of the flux densities to the uncertainties in quadrature to account for systematic uncertainties.

\section{FLUX DENSITIES FROM THE LITERATURE}

In this section we describe the procedure we followed to obtain previously recorded radio observations $(10 \mathrm{MHz}<$ $\nu<250 \mathrm{GHz}$ ) for all $30 z>4.5 \mathrm{VLBI}$ sources from the literature. These literature values are included with our observations (Section 2) to produce the final spectra in Section 4 .

For each source, we obtained the detected radio flux densities from the NASA/IPAC Extragalactic Database $(\mathrm{NED})^{3}$. Additionally, we recorded all unique matches to the source in the catalogues in the VizieR database (Ochsenbein et al. 2000) and in articles in the SAO/NASA Astrophysics Data System $(\mathrm{ADS})^{4}$. In each case, a matching radius of 20 arcsec from the VLBI position was used.

A number of our targets were observed, but not detected, in the following large surveys: The VLA LowFrequency Sky Survey Redux (VLSSr, $74 \mathrm{MHz}$; Lane et al. 2014), TGSS, WENSS, the Green Bank $4.85 \mathrm{GHz}$ survey (GB6, $4850 \mathrm{MHz}$; Gregory et al. 1996), the $62 \mathrm{MHz}$ LowFrequency Array (LOFAR) image of the Boötes field made

3 http://ned.ipac.caltech.edu/

4 http://adsabs.harvard.edu/ by Van Weeren et al. (2014) and the $3 \mathrm{GHz}$ Caltech-NRAO Stripe 82 Survey (CNSS; Mooley et al. 2016). To determine consistent upper limits for these non-detections, we downloaded the survey images and measured $\sigma_{\text {local }}$ within the $10 \times 10$ arcmin area surrounding the VLBI position. The flux density upper limit was then recorded as $3 \sigma_{\text {local }}$. As there were no images available for the GB6 survey, we used the detection threshold of $18 \mathrm{mJy}$ (Gregory et al. 1996) as an upper limit.

As we have known VLBI coordinates for our targets, we used a lower detection threshold ( $\left.3 \sigma_{\text {local }}\right)$ than the VLSSr, WENSS $\left(5 \sigma_{\text {local }}\right)$ and TGSS surveys $\left(7 \sigma_{\text {local }}\right)$. To include the $3 \sigma_{\text {local }}$ detections from these surveys, we ran source extraction on the survey images using PYBDSM as described in Section 2. The flux densities of sources that were detected at a significance (defined as the sources peak brightness divided by $\left.\sigma_{\text {local }}\right)$ greater than $3 \sigma_{\text {local }}$, and for which the source position differed by less than half the FWHM of the restoring beam of the image were recorded as detections. These detections are listed in Table 3. For these sources, the uncertainties on the $148 \mathrm{MHz}$ TGSS and $74 \mathrm{MHz}$ VLSSr flux densities were increased by 10 and 12 per cent, respectively, to account for systematic uncertainties, as was done in Intema et al. (2016) and Lane et al. (2014).

The observations and surveys have different angular resolutions, so we checked for possible blended sources. Using the $1.4 \mathrm{GHz}$ FIRST survey, we recorded the separation between each of our targets and their nearest neighbouring source. If the target was not in the $1.4 \mathrm{GHz}$ FIRST survey, we used TGSS $(148 \mathrm{MHz})$ or the $1.4 \mathrm{GHz}$ Sloan Digital Sky Survey (SDSS) STRIPE82 (Hodge et al. 2011) catalogue (which have resolutions of 25 and 1.8 arcsec, respectively) instead. For each of the detections we then checked whether the nearest neighbour could be distinguished from the target. All blended sources were discarded. These cases are discussed individually for each source in Section 4.

As a final step, we plotted each of the spectra (Section 4) and discarded the upper limits that were too high to valuably constrain the spectra. All upper limits that were used are given in Table 5 .

\section{RADIO SPECTRA}

In this section we will discuss each of the sources individually, and classify their spectra into one of the following classes: flat-spectrum sources, steep-spectrum sources, peaked-spectrum sources, and sources with unusual spectra (or spectra that could be classified into more than one class). A summary of the classification of each source is given in Table 4 .

Each flux density point in the spectra is labelled with the name of the survey, or else according to the following convention: the first characters are the initial letters of the surnames for the lead authors of the article in which the flux density was published. These characters are followed by the year of publication. If the flux density is from a VLBI observation, the year is followed by ' $(\mathrm{V})$ '. In the spectra (Figures 1, 2 and 5-26 ) VLBI flux densities are also shown as filled grey symbols to distinguish them from non-VLBI flux densities. Upper limits are indicated by an unfilled downward arrow originating at the symbol. We note that for some 
Table 2. GMRT image parameters

\begin{tabular}{|c|c|c|c|c|c|c|c|}
\hline \multirow{2}{*}{$\begin{array}{l}\text { ID } \\
\text { (1) }\end{array}$} & \multirow{2}{*}{$\begin{array}{l}\text { Observation } \\
\text { name } \\
(2)\end{array}$} & \multirow{2}{*}{$\begin{array}{c}\text { Flux density } \\
{[\mathrm{mJy}]} \\
(3)\end{array}$} & \multirow{2}{*}{$\begin{array}{c}\text { Local noise } \\
{\left[\mathrm{mJy}_{\text {beam }}^{-1}\right]} \\
\text { (4) }\end{array}$} & \multicolumn{2}{|c|}{ Deconvolved source size } & \multicolumn{2}{|c|}{ Restoring beam } \\
\hline & & & & $\begin{array}{c}{[\operatorname{arcsec}]^{\mathrm{a}}} \\
(5)\end{array}$ & $\begin{array}{c}\mathrm{PA}\left[{ }^{\circ}\right]^{\mathrm{b}} \\
(6)\end{array}$ & $\begin{array}{c}\text { arcsec }] \\
\quad(7)\end{array}$ & $\begin{array}{c}\mathrm{PA}\left[{ }^{\circ}\right] \\
(8)\end{array}$ \\
\hline \multirow[t]{4}{*}{ J0210-0018 } & GMRT610S & $10.5 \pm 1.1$ & 0.04 & $(1.1 \pm 0.1) \times(0.0 \pm 0.1)$ & $25 \pm 1$ & $7.1 \times 4.0$ & 100 \\
\hline & GMRT610N & $4.4 \pm 0.5$ & 0.04 & $(1.7 \pm 0.2) \times(0.0 \pm 0.1)$ & $55 \pm 2$ & $7.1 \times 4.0$ & 100 \\
\hline & GMRT325 & $19.0 \pm 2.1$ & 0.32 & $(0.0 \pm 0.1) \times(0.0 \pm 0.1)$ & $0 \pm 3$ & $9.3 \times 6.7$ & 63 \\
\hline & GMRT150 & $23.0 \pm 8.0$ & 4.50 & $(0.0 \pm 8.9) \times(0.0 \pm 2.5)$ & $0 \pm 16$ & $32.2 \times 16.7$ & 64 \\
\hline \multirow[t]{2}{*}{ J0940+0526 } & GMRT610 & $102.8 \pm 10.3$ & 0.09 & $(1.2 \pm 0.1) \times(0.9 \pm 0.1)$ & $115 \pm 1$ & $4.8 \times 4.0$ & 87 \\
\hline & GMRT325 & $135.1 \pm 13.6$ & 0.62 & $(2.2 \pm 0.1) \times(2.1 \pm 0.1)$ & $115 \pm 2$ & $10.1 \times 8.9$ & 0 \\
\hline \multirow[t]{4}{*}{$\mathrm{J} 1146+4037$} & GMRT610 & $6.8 \pm 0.7$ & 0.08 & $(0.8 \pm 0.1) \times(0.0 \pm 0.1)$ & $55 \pm 2$ & $5.8 \times 4.1$ & 112 \\
\hline & GMRT325 & $4.6 \pm 0.5$ & 0.05 & $(2.1 \pm 0.1) \times(0.9 \pm 0.1)$ & $18 \pm 2$ & $9.7 \times 7.4$ & 61 \\
\hline & GMRT235 & $4.9 \pm 1.1$ & 0.61 & $(0.0 \pm 2.9) \times(0.0 \pm 1.3)$ & $0 \pm 19$ & $14.7 \times 10.8$ & 113 \\
\hline & GMRT150 & $4.6 \pm 1.4$ & 0.73 & $(0.0 \pm 7.1) \times(0.0 \pm 1.9)$ & $0 \pm 13$ & $25.0 \times 16.8$ & 13 \\
\hline \multirow[t]{4}{*}{$\mathrm{J} 1242+5422$} & GMRT610 & $29.7 \pm 3.0$ & 0.10 & $(1.5 \pm 0.1) \times(0.8 \pm 0.1)$ & $129 \pm 1$ & $5.8 \times 4.1$ & 138 \\
\hline & GMRT325 & $30.0 \pm 3.0$ & 0.10 & $(1.2 \pm 0.1) \times(0.9 \pm 0.1)$ & $52 \pm 1$ & $10.8 \times 7.6$ & 45 \\
\hline & GMRT235 & $27.6 \pm 2.9$ & 0.56 & $(2.1 \pm 0.1) \times(0.0 \pm 0.1)$ & $47 \pm 3$ & $14.8 \times 10.7$ & 146 \\
\hline & GMRT150 & $26.1 \pm 2.9$ & 0.69 & $(0.0 \pm 0.8) \times(0.0 \pm 0.1)$ & $0 \pm 3$ & $27.2 \times 17.3$ & 1 \\
\hline \multirow[t]{2}{*}{$\mathrm{J} 1400+3149$} & GMRT610 & $24.6 \pm 2.5$ & 0.08 & $(0.9 \pm 0.1) \times(0.7 \pm 0.1)$ & $177 \pm 1$ & $4.6 \times 3.6$ & 51 \\
\hline & GMRT150 & $56.2 \pm 6.5$ & 2.11 & $(22.3 \pm 2.6) \times(9.9 \pm 0.9)$ & $63 \pm 5$ & $24.9 \times 15.6$ & 70 \\
\hline $\mathrm{J} 1548+3335$ & GMRT610 & $77.6 \pm 7.8$ & 0.19 & $(1.9 \pm 0.1) \times(1.3 \pm 0.1)$ & $66 \pm 1$ & $9.4 \times 4.0$ & 83 \\
\hline \multirow{2}{*}{$\mathrm{J} 1628+1154$} & GMRT610 & $107.7 \pm 10.8$ & 0.13 & $(1.9 \pm 0.1) \times(0.3 \pm 0.1)$ & $25 \pm 1$ & $6.0 \times 3.5$ & 82 \\
\hline & GMRT325 & $152.4 \pm 15.3$ & 0.63 & $(1.8 \pm 0.1) \times(0.5 \pm 0.1)$ & $171 \pm 1$ & $11.6 \times 7.1$ & 83 \\
\hline \multirow[t]{4}{*}{$\mathrm{J} 1659+2101$} & GMRT610 & $48.1 \pm 4.8$ & 0.13 & $(1.2 \pm 0.1) \times(0.5 \pm 0.1)$ & $73 \pm 1$ & $4.6 \times 3.6$ & 24 \\
\hline & GMRT325 & $53.0 \pm 5.3$ & 0.13 & $(3.0 \pm 0.1) \times(1.2 \pm 0.1)$ & $44 \pm 1$ & $10.2 \times 6.7$ & 65 \\
\hline & GMRT235 & $54.7 \pm 5.7$ & 0.84 & $(0.0 \pm 0.1) \times(0.0 \pm 0.1)$ & $0 \pm 3$ & $12.0 \times 9.5$ & 22 \\
\hline & GMRT150 & $48.2 \pm 5.4$ & 1.45 & $(8.4 \pm 0.9) \times(2.0 \pm 0.4)$ & $47 \pm 4$ & $21.6 \times 15.1$ & 17 \\
\hline
\end{tabular}

Columns: Col. 1 - source name (J2000); Col. 2 - observation name; Col. 3 -integrated flux densities and uncertainties; Col. 4 -rms noise at the source position; Col. 5 - deconvolved source size (FWHM); Col. 6 - deconvolved major axis position angle (measured from north through east); Col. 7 - Gaussian restoring beam size (FWHM); Col. 8 - Gaussian restoring beam major axis position angle (measured from north through east).

Notes: ${ }^{a}$ Uncertainties that would round down to zero are reported as 0.1 arcsec. ${ }^{b}$ Uncertainties that would round down to zero are reported as $1^{\circ}$.

Table 3. Flux densities of sources that are not in the survey catalogues but that were detected

\begin{tabular}{ccccc}
\hline ID & $\begin{array}{c}\text { Observation } \\
\text { name }\end{array}$ & $\begin{array}{c}\nu \\
{[\mathrm{MHz}]}\end{array}$ & $\begin{array}{c}\text { Flux density } \\
{[\mathrm{mJy}]}\end{array}$ & $\begin{array}{c}\text { Detection } \\
\text { significance } \\
{\left[\sigma_{\text {local }}\right]^{\mathrm{a}}}\end{array}$ \\
\hline J0131-0321 & TGSS & 148 & $24.6 \pm 4.5$ & $\sim 7.5$ \\
J0210-0018 & TGSS & 148 & $30.3 \pm 6.0$ & $\sim 6.6$ \\
J1026+2542 & VLSSr & 74 & $631 \pm 237$ & $\sim 4.1$ \\
J1628+1154 & VLSSr & 74 & $611 \pm 239$ & $\sim 4.3$ \\
\hline
\end{tabular}

Notes: ${ }^{a}$ The detection significance was calculated by dividing the source peak brightness by the local rms noise.

publications and catalogues, no flux density errors are available. This is the case for the PBW1992, B2.2 and B3 catalogues, however, following Vollmer et al. (2005), we assumed errors of 10 per cent for PBW1992 and 20 per cent for B2.2 and B3. A table containing all of the flux density labels, the observing frequency at which the measurement was taken, and the literature reference is given in Appendix A. A table containing the flux density values in the spectra of each source is given as online-only material. A sample of the table is shown in Table 5 .
Throughout this section, when fitting the spectra we used a linear least-squares fitting routine. Because of their much higher angular resolution, VLBI measurements are insensitive to the large-scale radio emission. VLBI flux densities are therefore usually underestimates of the total flux densities, unless the source is very compact. Consequently, unless specifically noted, the spectral fits do not include VLBI flux densities, flux densities without uncertainties and flux density upper limits. Note that the values in the spectra are integrated flux densities unless only the peak brightness was available. We finally point out that in most cases the flux density measurements used here are taken at different epochs. In the case of source variability, this may affect the estimated spectral index.

All of the sources have single components in their nonVLBI images unless noted otherwise in the discussion of the source. The VLBI morphological classifications of all of the sources are given in CFC2016.

\subsection{Flat-spectrum sources}

The following six sources all have flat spectra (they can be fitted by a single power law with $-0.5<\alpha<0.5$ ). 
Table 4. Summary of the spectral classification of each source

\begin{tabular}{|c|c|}
\hline ID & Classification $^{\mathrm{a}}$ \\
\hline J0011+1446 & Flat \\
\hline J0131-0321 & Flat \\
\hline J0210-0018 & Flat (steep) \\
\hline $\mathrm{J} 0311+0507$ & Steep (USS) \\
\hline J0324-2918 & Peaked \\
\hline $\mathrm{J} 0813+3508$ & Steep \\
\hline J0836+0054 & Steep (USS) \\
\hline J0906+6930 & Peaked \\
\hline J0913+5919 & Peaked \\
\hline J0940+0526 & Steep \\
\hline $\mathrm{J} 1013+2811$ & Flat or peaked \\
\hline $\mathrm{J} 1026+2542$ & Flat \\
\hline J1146+4037 & Peaked (inverted) \\
\hline J1205-0742 & Concave \\
\hline J1235-0003 & Peaked \\
\hline $\mathrm{J} 1242+5422$ & Peaked \\
\hline $\mathrm{J} 1311+2227$ & Inverted or flat or peaked \\
\hline $\mathrm{J} 1400+3149$ & Flat \\
\hline $\mathrm{J} 1427+3312$ & Steep (flat) \\
\hline $\mathrm{J} 1429+5447$ & Steep \\
\hline $\mathrm{J} 1430+4204$ & Flat \\
\hline J1454+1109 & Unknown \\
\hline J1548+3335 & Steep \\
\hline $\mathrm{J} 1606+3124$ & Peaked \\
\hline $\mathrm{J} 1611+0844$ & Inverted or flat or peaked \\
\hline J1628+1154 & Steep \\
\hline $\mathrm{J} 1659+2101$ & Peaked \\
\hline $\mathrm{J} 1720+3104$ & Flat or peaked \\
\hline $\mathrm{J} 2102+6015$ & Peaked \\
\hline $\mathrm{J} 2228+0110$ & Peaked \\
\hline
\end{tabular}

Notes: a Wording such as 'Flat (steep)' indicates that the source has a flat spectral index, but that it could be steep within the uncertainties. Wording such as 'Flat or peaked' is used when there is insufficient information to classify the spectrum of the source, but (often using upper limits) it is possible to exclude certain spectral types.

\subsubsection{J0011+1446}

We matched J0011+1446 to sources in the $148 \mathrm{MHz}$ TGSS, National Radio Astronomy Observatory (NRAO) VLA Sky Survey (NVSS; Condon et al. 1998) and $4.9 \mathrm{GHz}$ GB6 catalogues. However, in the $1.4 \mathrm{GHz}$ FIRST catalogue there are two sources that are 16.4 and 29.3 arcsec away from the J0011+1446 VLBI position. Since the flux density of these sources will blend with that of J0011+1446 in the lower resolution TGSS, $1.4 \mathrm{GHz}$ NVSS and GB6 catalogues, we discarded these matches. The spectrum is shown in Fig. 1. Fitting a power law between the FIRST and $8.5 \mathrm{GHz}$ CLASS flux densities gives a spectral index of $\alpha=-0.25 \pm 0.11$. $\mathrm{J} 0011+1446$ is therefore a flat-spectrum source, although, because non-VLBI flux densities are only available at two frequencies, it is possible that it could also have a peaked or concave spectrum. From the spectrum it is clear that some of the source's flux density was resolved out in the VLBI observations, or the source is variable.

\subsubsection{J0131-0321}

A power-law fit for the spectrum of J0131-0321 (Fig. 2) gives $\alpha=0.12 \pm 0.10$. J0131-0321 is therefore a flatspectrum source, although, because non-VLBI flux densi-

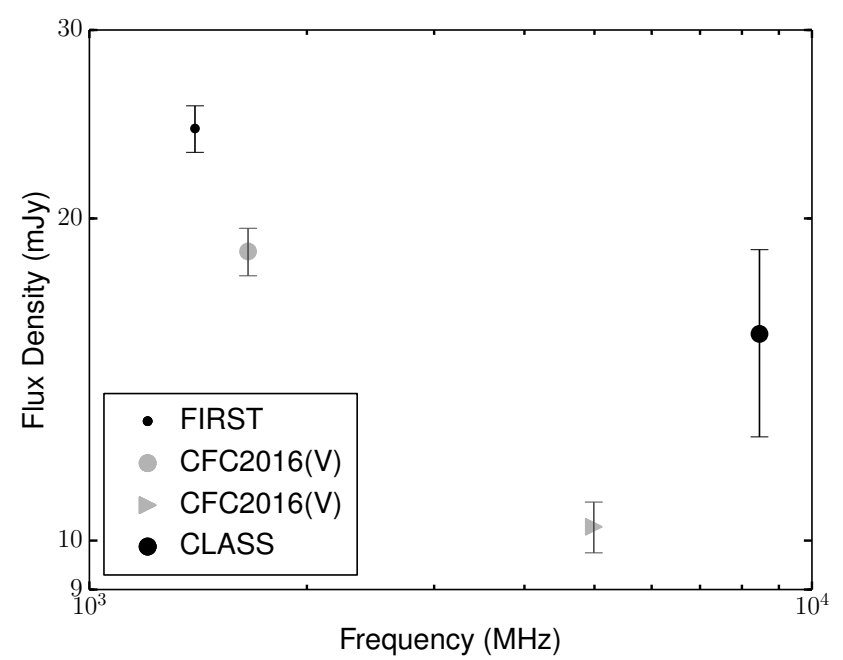

Figure 1. The radio spectrum of J0011+1446.

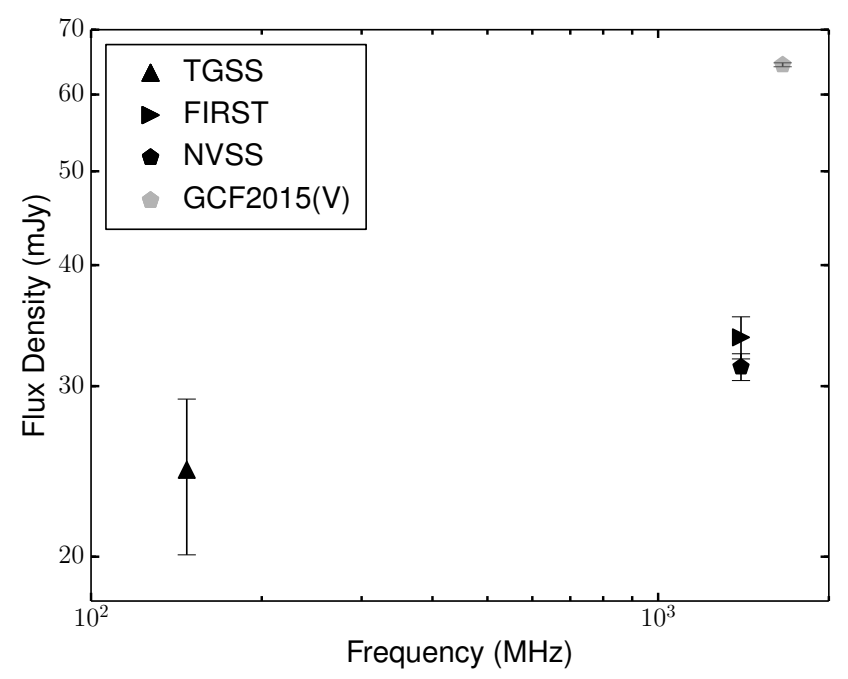

Figure 2. The radio spectrum of J0131-0321.

ties are only available at two frequencies, it is possible that it could also have a peaked or concave spectrum. GCF2015(V) observed this source with the EVN at $1.7 \mathrm{GHz}$ and found it to be unresolved, with a flux density of $64.4 \pm 0.3 \mathrm{mJy}$. Comparing this to the $1.4 \mathrm{GHz}$ FIRST and NVSS flux densities of $33.7 \pm 1.7$ and $31.4 \pm 1.0 \mathrm{mJy}$, respectively, GCF2015(V) concluded that J0131-0321 is likely variable. However, since the epochs when FIRST and NVSS observed J0131-0321 differ by about 15.25 years (Ofek \& Frail 2011; Helfand et al. 2015), if J0131-0321 is variable it means that the FIRST and NVSS observations were serendipitously done on two epochs when J0131-0321 happened to have the same flux density. The argument that J0131-0321 is variable is, however, supported by our finding that J0131-0321 has a flat spectrum, and GCF2015(V)'s conclusion that the VLBI emission is Doppler-boosted. 
Table 5. Example entries in the online-only table containing the flux density values for each source

\begin{tabular}{lccccc}
\hline Source name & Observation name & $\nu[\mathrm{MHz}]$ & Upper limit ${ }^{\mathrm{a}}$ & $\begin{array}{c}\text { Flux density } \\
{[\mathrm{mJy}]}\end{array}$ & $\begin{array}{c}\text { Flux density } \\
\text { error [mJy] }\end{array}$ \\
\hline J0011+1446 & FIRST & 1400 & $\mathrm{~N}$ & 24.3 & 1.2 \\
J0011+1446 & CFC2016(V) & 1658 & $\mathrm{~N}$ & 18.6 & 1.0 \\
J0011+1446 & CFC2016(V) & 4990 & $\mathrm{~N}$ & 10.3 & 0.6 \\
J0011+1446 & CLASS & 8460 & $\mathrm{~N}$ & 15.6 & 3.1 \\
J0131-0321 & TGSS & 148 & $\mathrm{~N}$ & 24.6 & 4.5 \\
J0131-0321 & FIRST & 1400 & $\mathrm{~N}$ & 33.7 & 1.7 \\
J0131-0321 & NVSS & 1400 & $\mathrm{~N}$ & 31.4 & 1.0 \\
J0131-0321 & GCF2015(V) & 1658 & $\mathrm{~N}$ & 64.4 & 0.3 \\
\hline
\end{tabular}

Notes: " $Y$ " indicates that the value is an upper limit, "N" indicates that the value is not an upper limit.

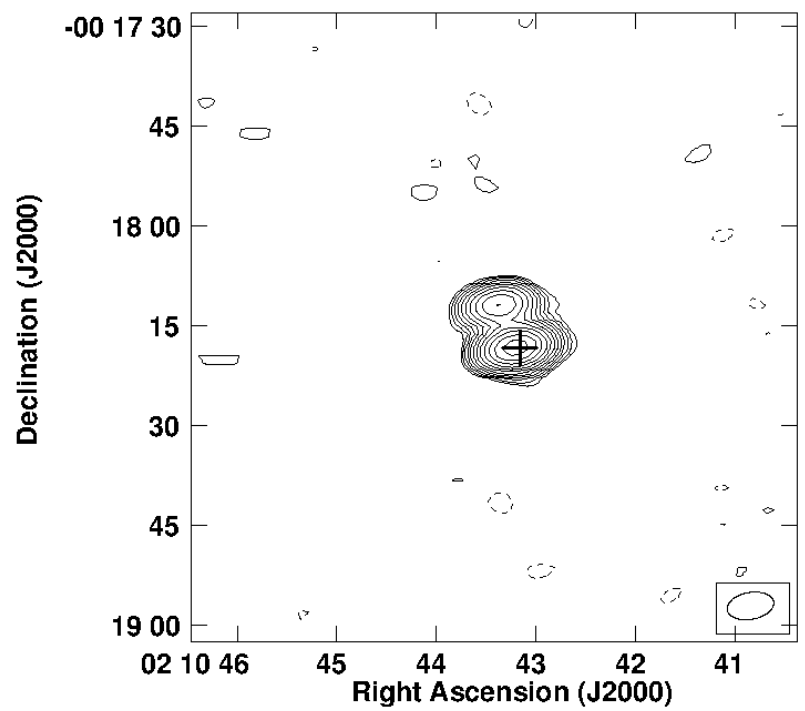

Figure 3. $608 \mathrm{MHz}$ GMRT610 image of J0210-0018. The lowest contours are drawn at -0.18 and $0.18 \mathrm{mJy}$ beam $^{-1}$, the positive contours increase in factors of $\sqrt{2}$ thereafter. The restoring beam (FWHM) is shown in the bottom right corner and the position of the optical AGN is indicated by a cross.

\subsubsection{J0210-0018}

Fig. 3 and 4 show the $608 \mathrm{MHz}$ GMRT610 and $1.4 \mathrm{GHz}$ VLA STRIPE82 images of J0210-0018. In both of these images the source has two components. Table 6 gives the flux densities of the individual components. Using the GMRT610 and STRIPE82 flux densities we calculate spectral indices of $-0.79 \pm 0.21$ and $-0.36 \pm 0.13$ for the northern and southern components, respectively.

In all the other observations (except for the $1.4 \mathrm{GHz}$ FIRST observations), J0210-0018 only has a single component due to a lack of resolution. Although FIRST has sufficient resolution to resolve J0210-0018, the source is fit by a single component with deconvolved major and minor axes of 4.3 and 1.3 arcsec, respectively. The FIRST image does show an indication of a second component at the position of the northern component. It is not detected however, because the separation between the two components is small, and the

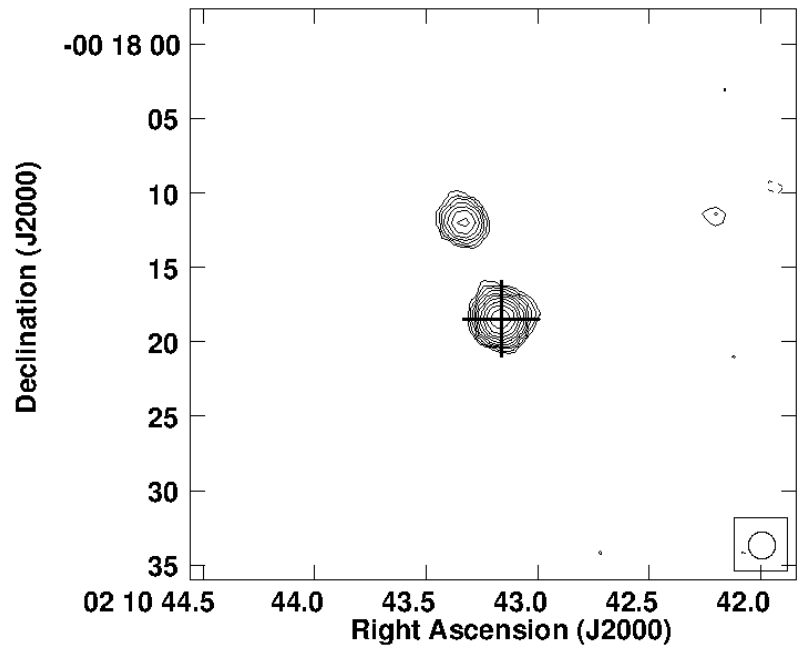

Figure 4. $1.4 \mathrm{GHz}$ STRIPE82 image of J0210-0018. The lowest contours are drawn at -0.21 and $0.21 \mathrm{mJy}$ beam $^{-1}$. The positive contours increase in factors of $\sqrt{2}$ thereafter. The restoring beam (FWHM) is shown in the bottom right corner and the position of the optical AGN is indicated by a cross.

Table 6. J0210-0018 component flux densities

\begin{tabular}{ccc}
\hline Image & Component & $\begin{array}{c}\text { Flux density } \\
{[\mathrm{mJy}]}\end{array}$ \\
\hline GMRT610 & north & $4.36 \pm 0.45$ \\
& south & $10.46 \pm 1.05$ \\
STRIPE82 & north & $2.22 \pm 0.33$ \\
& south & $7.72 \pm 0.34$ \\
\hline
\end{tabular}

northern component is significantly fainter than the southern component. At $1.4 \mathrm{GHz}$ the two components are therefore only detected in the STRIPE82 catalogue, which has both higher resolution and sensitivity than FIRST. Using the STRIPE82 positions of the two components, the angular separation between the components is 7.0 arcsec, which translates to a linear separation of $\sim 45.6 \mathrm{kpc}$.

The southern component coincides positionally with the optical AGN (Figures 3 and 4). In principle there are four 
possibilities for what J0210-0018 could be: (1) the two components are unrelated sources at different redshifts; (2) the northern and southern components are gravitationally lensed images of the same source; (3) J0210-0018 is a onesided source where one of the components is a hotspot or a lobe of the other; (4) the two components are separate, unrelated AGN at the same redshift.

The possibility that the two components of J0210-0018 are formed by gravitational lensing is unlikely given that the southern component positionally coincides with the optical AGN. In addition, if they are formed by gravitational lensing, the two components will have the same radio spectral index, which is not the case. We therefore conclude that the components are not gravitationally lensed images of the same source. One way to confirm that the two components are related is to search for a jet between them. Using our previous 1.7 and $5 \mathrm{GHz}$ EVN observations of J0210-0018 (Coppejans et al. 2016), in which the southern component was detected at both frequencies, we searched for a jet and did not find anything. We do, however, note that the $1.7 \mathrm{GHz}$ EVN flux density is only 22 per cent of the $1.4 \mathrm{GHz}$ STRIPE82 flux density of the southern component. This indicates that the VLBI observations resolved out a significant fraction of the source's flux density. Consequently it is possible that this flux density is contained in a jet between the components that was resolved out. This possibility is further supported by the fact that the southern and northern components have flat and steep spectra, respectively. This likely indicates that the southern component is the AGN core (which will have a flat spectrum), and the northern component is a lobe or a hotspot (which typically have steep spectra) in the southern component's jet. This interpretation is also supported by there being no optical counterpart to the northern component in the co-add of SDSS Stripe 82 imaging data (Abazajian et al. 2009), which reach a typical depth of $m_{r} \approx 24.5$ (Jiang et al. 2014).

In Fig. 5 we show the spectrum of J0210-0018. In the spectrum, the GMRT610 and STRIPE82 flux densities are the sums of the flux densities of the two components. Fig. 5 is therefore the sum of the spectra of both components. A power law fit to the spectrum gives $\alpha=-0.49 \pm 0.07$. We therefore classify J0210-0018 as having an overall flat spectrum. We do, however, note that J0210-0018 can be a steep-spectrum source (defined in Section 4.2) within the uncertainties.

\section{$4.1 .4 \quad J 1026+2542$}

We fitted the spectrum of J1026+2542 (Fig. 6) with a single power law with a spectral index of $\alpha=-0.41 \pm 0.02$. This is consistent with the value of $\alpha=-0.4$ found by FFP2013(V), and the fact that the source is Doppler-boosted (Coppejans et al. 2016).

\section{$4.1 .5 \quad J 1400+3149$}

We fitted the spectrum of J1400+3149 (Fig. 7) with a power law with a spectral index of $-0.36 \pm 0.07$.

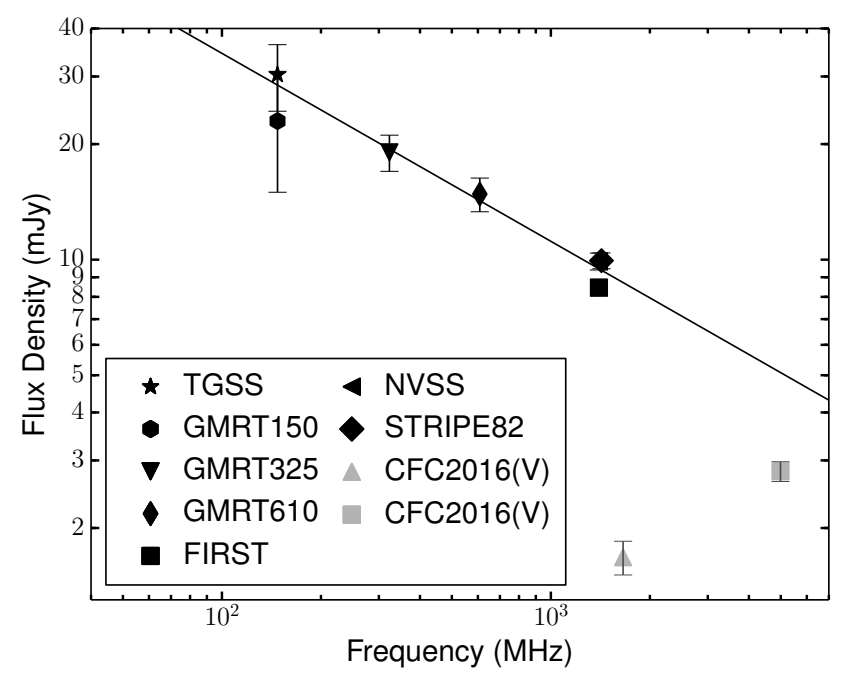

Figure 5. The radio spectrum of J0210-0018. The fit to the spectrum is shown as a solid line.

\subsubsection{J1430+4204}

WFP2006 observed J1430+4204 at $15.2 \mathrm{GHz}$ over a period of $\sim 7.5$ years, during which time they found the flux density to vary between $\sim 70$ and $\sim 430 \mathrm{mJy}$. Based on these findings and the spectrum of J1430+4204 (Fig. 8), we conclude that J1430+4204 is extremely variable. Fig. 8 gives the average 15.2 GHz WFP2006 flux density. Fitting a power law to the spectrum, we find a spectral index of $0.10 \pm 0.03$. While this spectral index is likely not a good indication of the spectral index of the source at any given time, it can be considered as an average spectral index. Combining this with the finding that J1430+4204 is Doppler-boosted (Coppejans et al. 2016), we conclude that J1430+4204 is a flat-spectrum radio quasar.

\subsection{Steep-spectrum and ultra-steep-spectrum sources}

The eight sources discussed in this section are all fitted with a single power-law spectrum with $\alpha<-0.5$. Included in this class of sources are the USS sources, which we will define as objects with $\alpha<-1.0$ across their entire spectral range.

\subsubsection{J0311+0507}

Matching the VLBI position for J0311+0507 to FIRST $(1.4 \mathrm{GHz})$, we find that there are 15 sources within two arcminutes of the source and that the nearest neighbour is 5.2 arcsec away. In the survey catalogue these sources are indicated to have side lobe probabilities between 0.063 and 0.528 (Helfand et al. 2015). Looking at the image of J0311+0507 in FIRST, the VLA beam pattern is clearly visible around the source, with the neighbouring sources all lying on the beam pattern ${ }^{5}$. Comparing the $1.4 \mathrm{GHz}$ FIRST and NVSS images and based on the probabilities of the

${ }^{5}$ http://third.ucllnl.org/cgi-bin/firstcutout 


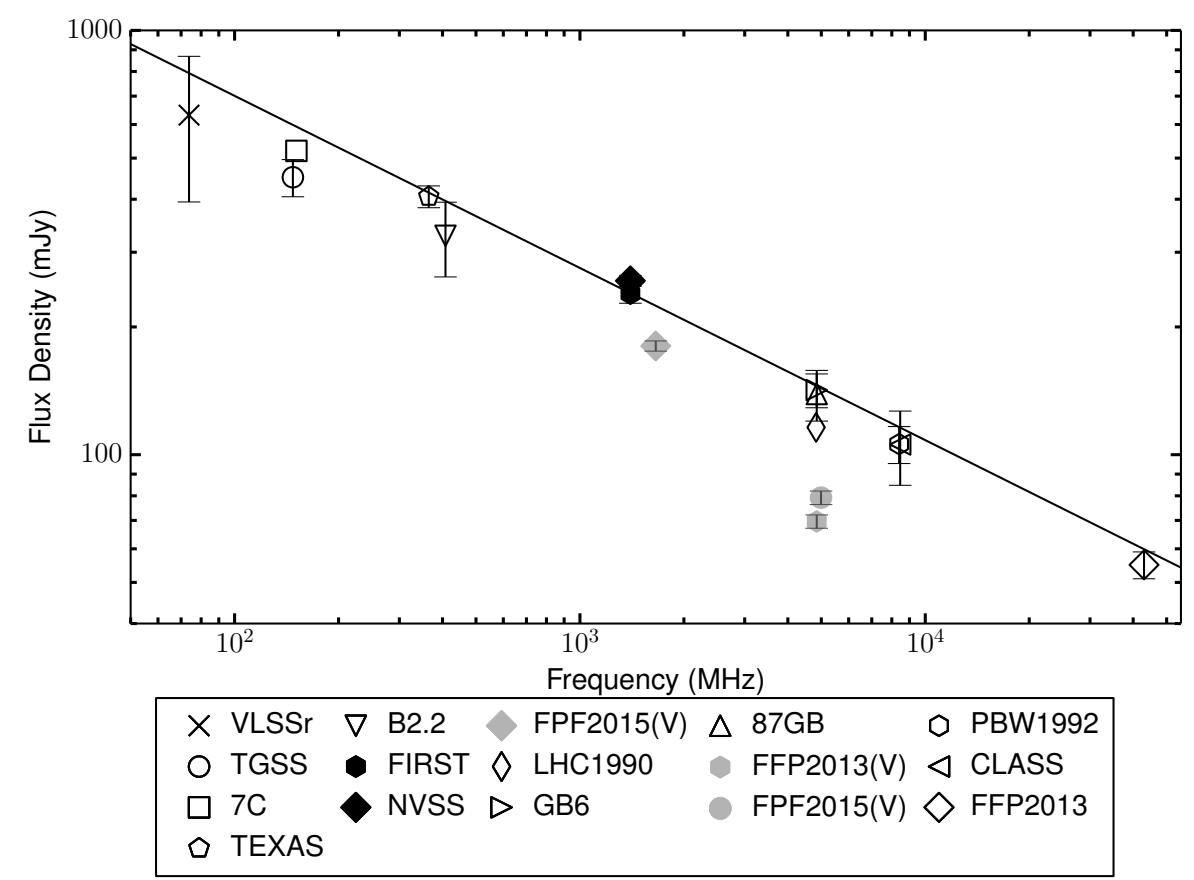

Figure 6. The radio spectrum of J1026+2542. The fit to the spectrum is shown as a solid line.

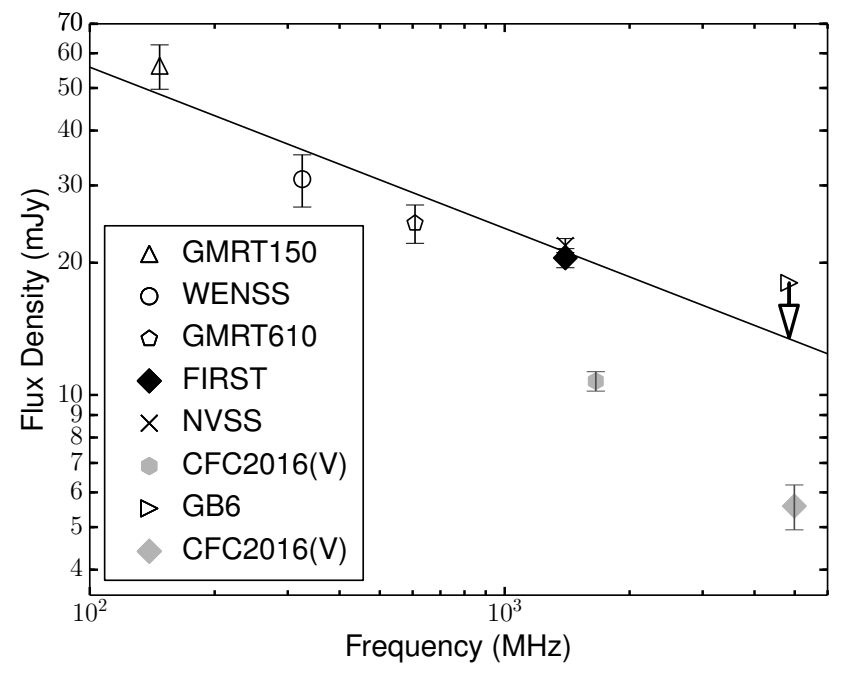

Figure 7. The radio spectrum of J1400+3149. The fit to the spectrum is shown as a solid line.

sources being side lobes, we conclude that the nearest real source to J0311+0507 is 330 arcsec away, and that the 15 neighbouring sources in FIRST are all artefacts. We matched $\mathrm{J} 0311+0507$ to the source $4 \mathrm{C}+04.11$ in the $178 \mathrm{MHz} 4 \mathrm{C}$ survey (Gower et al. 1967). However, because the $4 \mathrm{C}$ survey has a resolution of 11.5 arcmin, the flux density of the nearby sources will blend with that of J0311+0507, we discarded the match. We, for the same reason, discarded the matches to Bursov (1996) (at 0.96, 2.3, 3.94 and $7.69 \mathrm{GHz}$ ), Parijskij et al. (2010) (at 0.5, 1.4 and $3.94 \mathrm{GHz}$ ), Parijskij et al. (1996) (at 1.425 GHz), Pariiskii et al. (1992) (at $3.945 \mathrm{GHz}$ ) and Braude et al. (1979) (at $16.7 \mathrm{MHz}$ ).
J0311+0507 was classified as a USS source by Röettgering et al. (1994) who found it to have a spectral index of $-1.17 \pm 0.03$ between $150 \mathrm{MHz}$ and $4.85 \mathrm{GHz}$. We fitted the spectrum (shown in Fig. 9) with a single power law with a spectral index of $\alpha=-0.94 \pm 0.06$, and therefore classify J0311+0507 as a steep-spectrum source that could also be a USS source. We do note that our spectral index is higher than the spectral index of -1.31 between 365 and $4850 \mathrm{MHz}$ found by Goss et al. (1992) and Parijskij et al. (2014, and references therein). As a final point, we note that the 1.7 and $5 \mathrm{GHz}$ PTK2014(V) VLBI observations of J0311+0507 showed that it has a FR II structure, and an angular and linear size of 2.8 arcsec and $18.7 \mathrm{kpc}$, respectively.

\subsubsection{J0813+3508}

In FIRST $(1.4 \mathrm{GHz})$ there is a second source due northwest of the source matched to J0813+3508 that is 6.9 arcsec distant from the J0813+3508 VLBI position, which translates to a linear size of $\sim 43.7 \mathrm{kpc}$. FPG2010(V) observed both sources with the EVN at 1.7 and $5 \mathrm{GHz}$. While the second source was not detected, the authors did find a jet pointing from J0813+3508 towards the second source in the 1.7 GHz image. From this, FPG2010(V) concluded that the second source is a lobe of $\mathrm{J} 0813+3508$ that is resolved out by the VLBI observations. The only non-VLBI observation that has high enough resolution to resolve the two components is FIRST, in which the main and second components have flux densities of $37.5 \pm 1.9$ and $11.5 \pm 0.6 \mathrm{mJy}$, respectively. In the source spectrum (shown in Fig. 10), the FIRST flux density is therefore the sum of the flux densities of the two components. Fitting a power law to the spectrum we find $\alpha=-0.80 \pm 0.12$. We note that $148 \mathrm{MHz}$ TGSS has a resolution of $25 \times 25$ arcsec and that J0813+3508 has a fitted source size of $(28.8 \pm 1.4) \times(18.8 \pm 0.6) \operatorname{arcsec}$ in the 


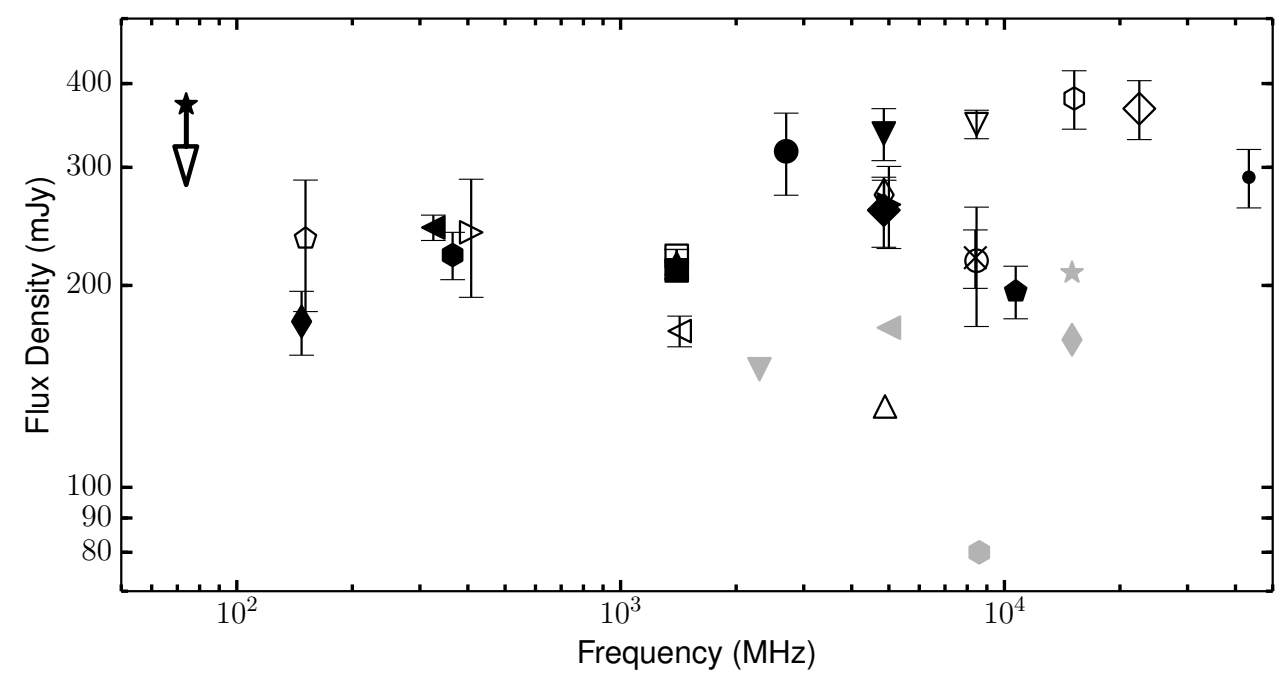

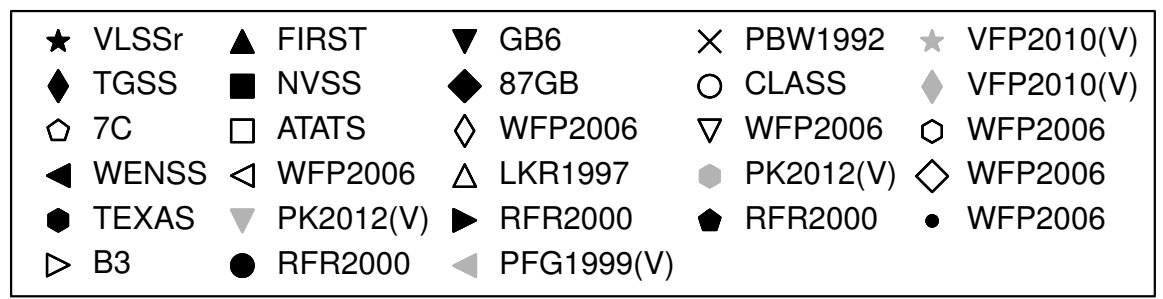

Figure 8. The radio spectrum of J1430+4204.

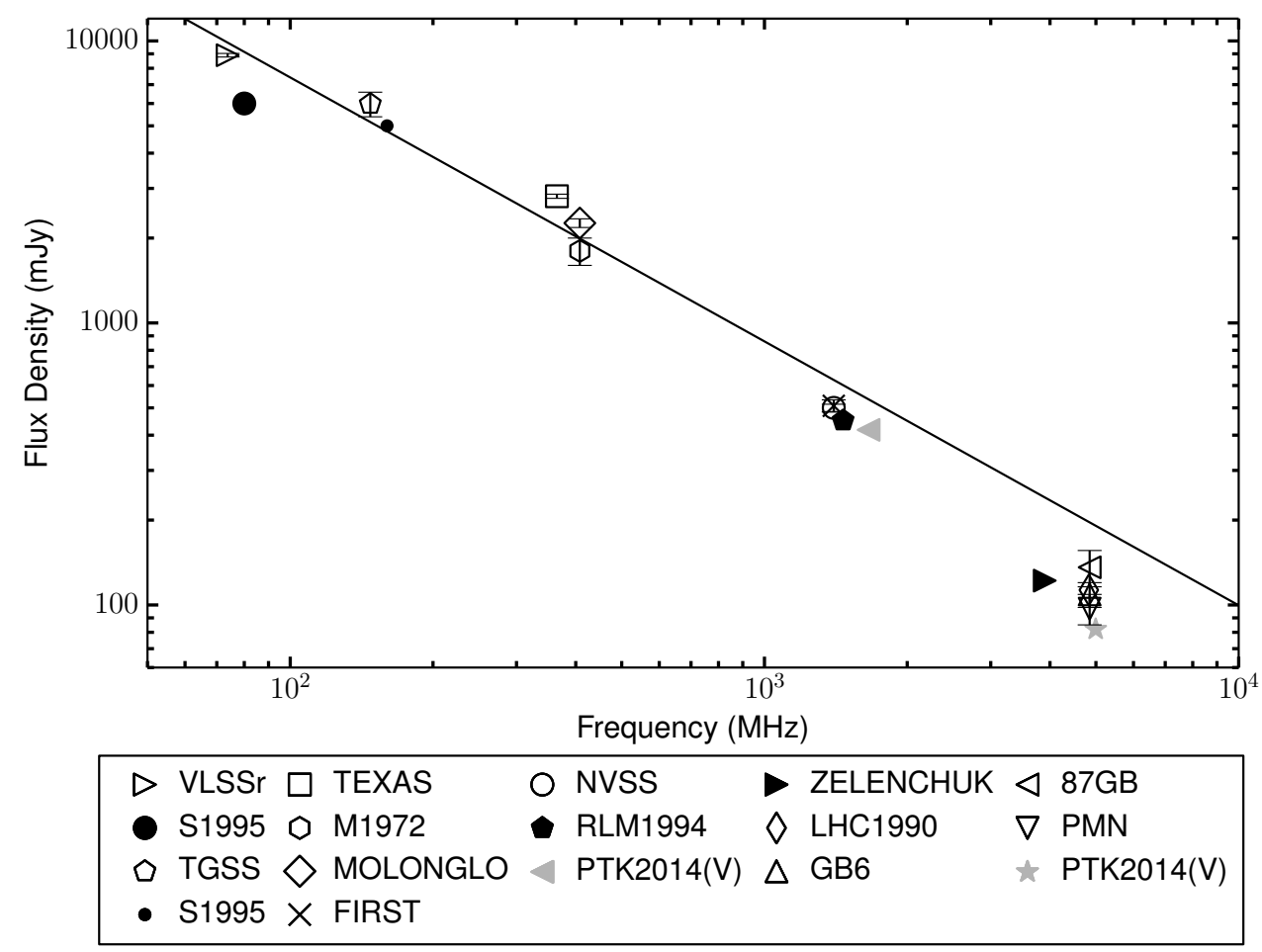

Figure 9. The radio spectrum of J0311+0507. The fit to the spectrum is shown as a solid line. 


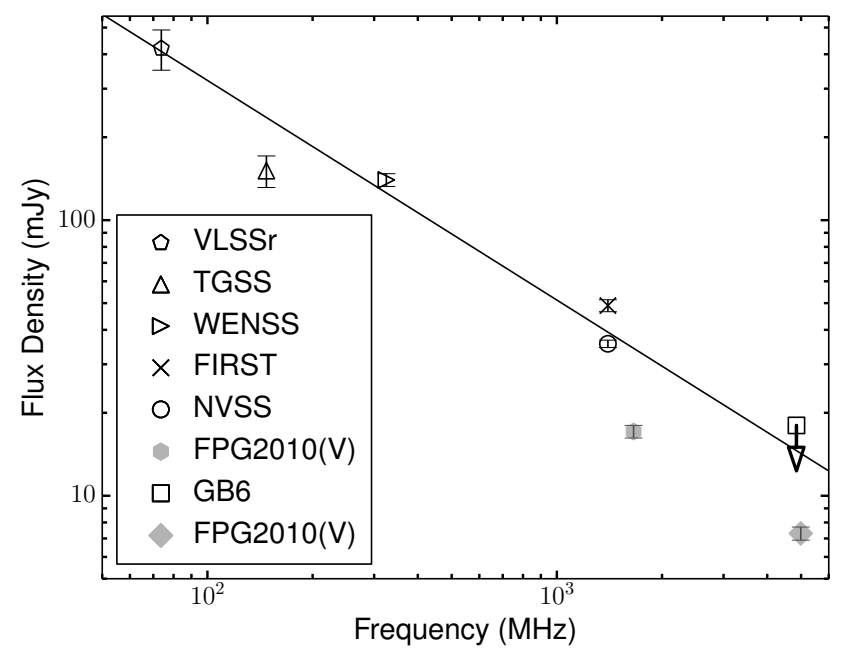

Figure 10. The radio spectrum of J0813+3508. The fit to the spectrum is shown as a solid line.

survey (Intema et al. 2016). The TGSS flux density being lower than the predicted value can therefore be explained by J0813+3508 being partially resolved or by variability.

\section{$4.2 .3 \quad J 0836+0054$}

Fitting the spectrum of J0836+0054 (Fig. 11) with a power law gives a spectral index of $\alpha=-0.89 \pm 0.29$. This indicates that the source can be a USS source within the uncertainties. J0836+0054 has $1.4 \mathrm{GHz}$ FIRST and NVSS flux densities of $1.11 \pm 0.06 \mathrm{mJy}$ and $2.5 \pm 0.5 \mathrm{mJy}$, respectively. In addition, PCB2003 found a $1.4 \mathrm{GHz}$ flux density of $1.75 \pm 0.04 \mathrm{mJy}$ during their observations with the VLA at a resolution of 1.5 arcsec. Since the PCB2003 observations have a higher resolution than FIRST, and $\mathrm{a} \sim 60$ per cent higher flux density, this, along with the flux density difference between FIRST and NVSS, could indicate that J0836+0054 is variable. However, the NVSS source is positionally offset from the FIRST source by about 15 arcsec to the northeast. Since NVSS has a resolution of 45 arcsec compared to the 5 arcsec of FIRST, the flux density and positional difference could also be because of resolution effects. This interpretation is supported by the PCB2003 flux density being consistent with the NVSS value and the PCB2003 observations having a $1 \sigma$ noise level of $0.0216 \mathrm{mJy}_{\text {beam }}{ }^{-1}$ compared to the $0.15 \mathrm{mJy}_{\text {beam }}{ }^{-1}$ of FIRST. Additionally the $1.4 \mathrm{GHz}$ FPM2005 flux density is consistent with both the NVSS and PCB2003 values but not with the FIRST value. While the FPM2005 observations have a resolution of $6.3 \times 4.4$ arcsec, which is similar to FIRST, they have a lower noise level of $0.083 \mathrm{mJy}$ beam $^{-1}$. We therefore conclude that J0836+0054 is likely not variable, but cannot rule out the possibility.

We finally note that the fitted spectrum predicts a $148 \mathrm{MHz}$ flux density of $\sim 12.0 \mathrm{mJy}$, while the $148 \mathrm{MHz}$ TGSS upper limit indicates that the flux density is below $6.1 \mathrm{mJy}$. This could be due to the uncertainty introduced in the fitted spectral index by the resolution effects mentioned above, variability, or a potential spectral turnover.

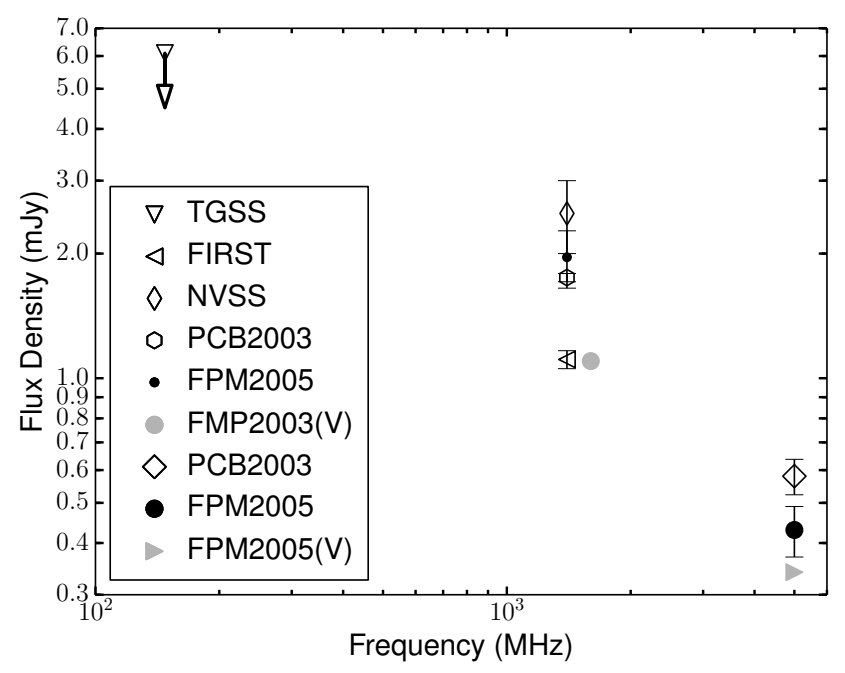

Figure 11. The radio spectrum of J0836+0054.

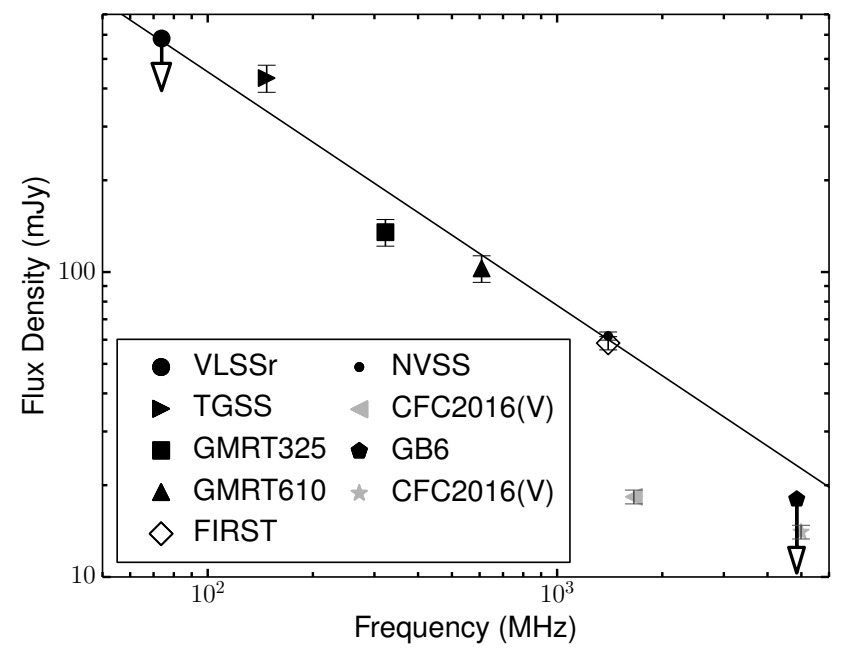

Figure 12. The radio spectrum of J0940+0526. The fit to the spectrum is shown as a solid line.

\subsubsection{J0940+0526}

We fitted the spectrum of J0940+0526 (Fig. 12) with a single power law with a spectral index of $\alpha=-0.77 \pm 0.10$.

\subsection{5 $31427+3312$}

We fitted the spectrum of J1427+3312 (Fig. 13) with a single power law with $\alpha=-0.62 \pm 0.17$. Although we classify the source as having a steep spectrum, it is also possible that it has a flat spectrum within the errors. Take note that the reason why the fitted line does not fit the $8.4 \mathrm{GHz}$ MCM2008 point very well is because the smaller errors on the $149 \mathrm{MHz}$ WWR2016 and $1.4 \mathrm{GHz}$ CMM1999 flux densities give these points larger weights during the fitting. Finally, we also note that the $1.4 \mathrm{GHz}$ FIRST and CMM1999 flux densities differ $(1.03 \pm 0.05$ and $1.82 \pm 0.02 \mathrm{mJy}$, respectively), and the 1.6 GHz FGP2008(V) and 1.4 GHz MCM2008(V) flux den- 


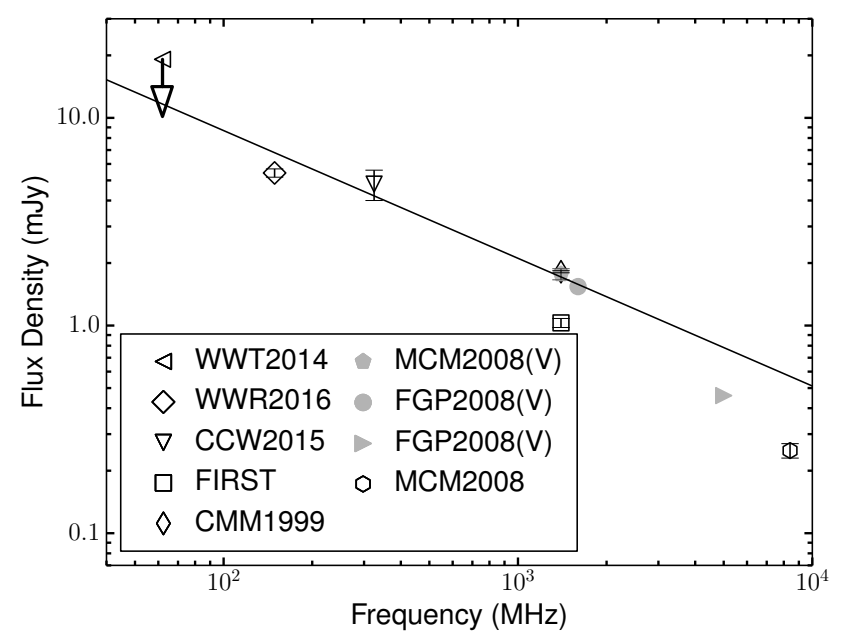

Figure 13. The radio spectrum of $\mathrm{J} 1427+3312$. The fit to the spectrum is shown as a solid line.

sities are higher than the FIRST flux density. The difference between the FIRST and CMM1999 flux densities could be caused by the CMM1999 observations having a resolution of $\sim 15$ arcsec, which is about three times lower than that of FIRST. The difference, specifically between FIRST and the VLBI flux densities, could also indicate that J1427+3312 is variable.

\subsubsection{J1429+5447}

OWB2013 and CFC2016 concluded that in the spectrum of $\mathrm{J} 1429+5447$ (Fig. 14) the emission below $100 \mathrm{GHz}$ is from AGN activity. WWC2011 found that the CO line emission of the source is resolved into two components that are separated by $1.2 \operatorname{arcsec}(\sim 6.9 \mathrm{kpc})$, with the optical and continuum source positions being consistent with the western peak. The authors also note that the eastern component is possibly extended with a size of $(1.1 \pm 0.2) \times(0.7 \pm 0.2)$ arcsec, which could explain why it is not detected in the continuum observations. OWB2013 also observed J1429+5447 at $250 \mathrm{GHz}$ and concluded that the majority of the $250 \mathrm{GHz}$ emission is thermal emission from hot dust. The authors do however note that it is possible that a significant fraction of the $250 \mathrm{GHz}$ emission could be from AGN driven synchrotron emission. Excluding the $250 \mathrm{GHz}$ OWB2013 value and fitting the spectrum with a power law gives $\alpha=-0.79 \pm 0.04$. We therefore classify $\mathrm{J} 1429+5447$ as a steep-spectrum source.

\subsubsection{J1548+3335}

We fitted a power law to the spectrum of $\mathrm{J} 1548+3335$ (Fig. 15) with a spectral index of $\alpha=-0.64 \pm 0.05$. We note that the $74 \mathrm{MHz}$ VLSSr and $4.9 \mathrm{GHz}$ GB6 upper limits could indicate that the spectrum is peaked. However, because there is an equal probability that the flux density of the source is at any value below (including only slightly below) the upper limits, additional observations are required to confirm or refute this.

In the $1.7 \mathrm{GHz}$ EVN observations, J1548+3335 was

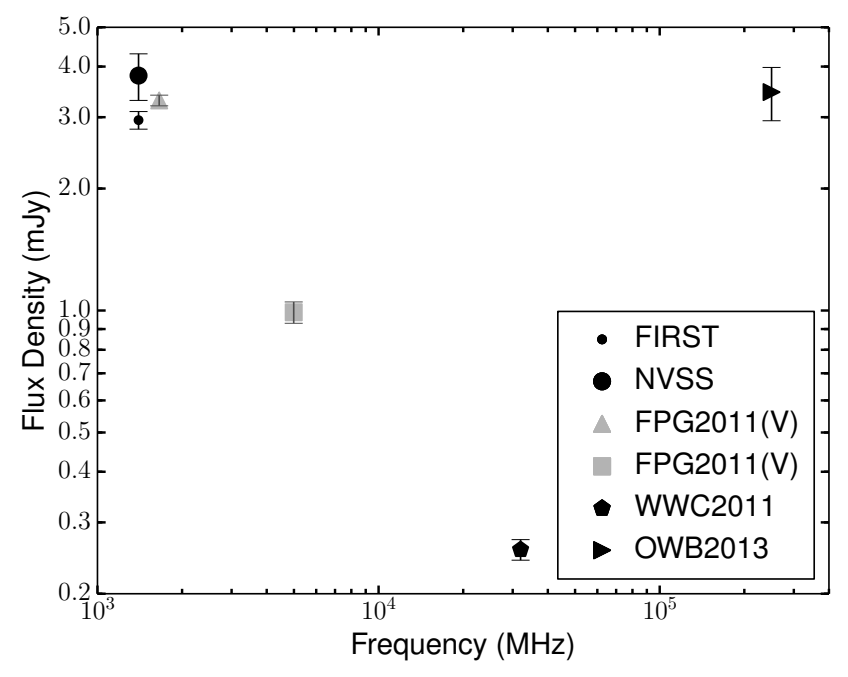

Figure 14. The radio spectrum of J1429+5447.

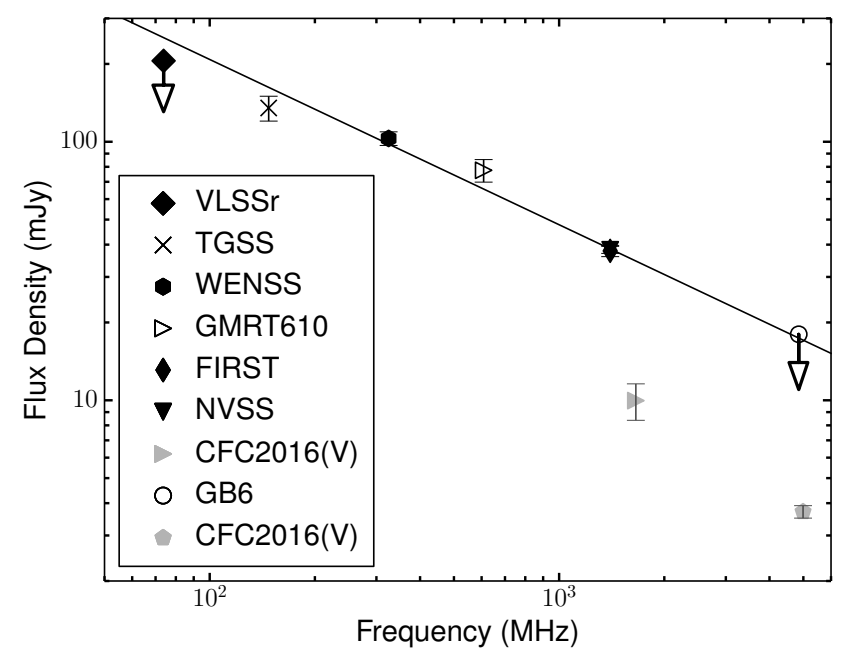

Figure 15. The radio spectrum of J1548+3335. The fit to the spectrum is shown as a solid line.

found to have two components that are separated by $812 \pm 3$ mas, which translates to a projected linear size of $5267 \pm 17$ pc (Coppejans et al. 2016). The second (fainter) component is not detected in the $5 \mathrm{GHz}$ EVN observations (Coppejans et al. 2016). The primary component coincides with the SDSS position and no jet was detected between the two components. It is, therefore, possible that the second component is a lobe or hotspot of the first component, an unrelated AGN at the same redshift, a foreground or background source that is unrelated to J1548+3335, or that the two components are gravitationally lensed images of the same source (Coppejans et al. 2016). From the spectrum it is clear that some of the source's flux density was resolved out in the $1.7 \mathrm{GHz}$ CFC2016(V) observations, or the source is variable. 


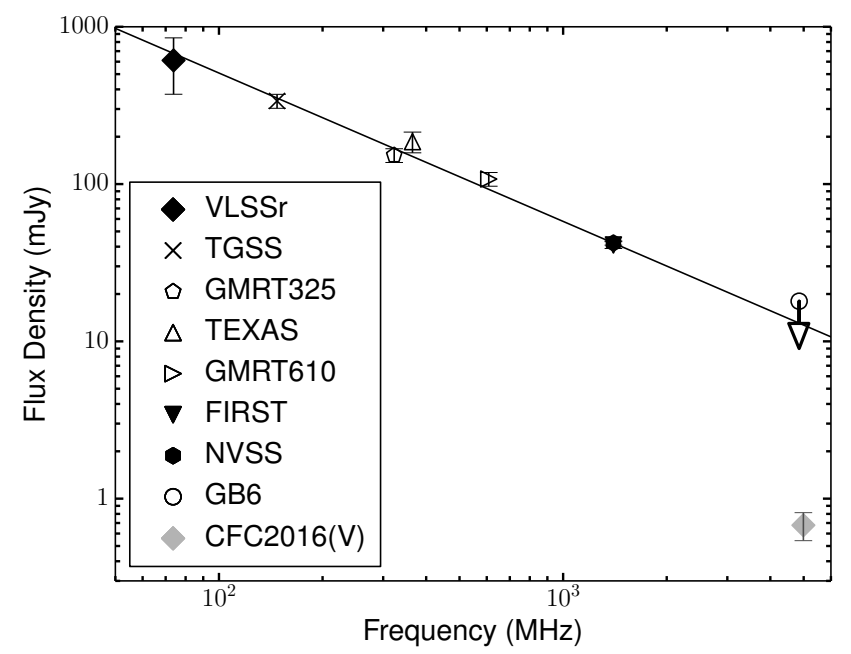

Figure 16. The radio spectrum of J1628+1154. The fit to the spectrum is shown as a solid line.

\subsubsection{J1628+1154}

We fitted the spectrum of J1628+1154 (Fig. 16) with a power law with $\alpha=-0.94 \pm 0.04$.

\subsection{Peaked-spectrum sources}

The following ten sources all have peaked spectra. Where appropriate, and following Orienti et al. (2007), Scaife \& Heald (2012) and Orienti \& Dallacasa (2014), we fitted the spectra with log parabolas of the form $\log _{10}(S)=$ $a\left[\log _{10}(\nu)-\log _{10}\left(\nu_{\mathrm{o}}\right)\right]^{2}+b$, where $a$ and $b$ are constants and $S$ is flux density.

\subsubsection{J0324-2918}

There is a discrepancy between the 4.8 and $8.6 \mathrm{GHz}$ AT20G flux densities, and the $8.4 \mathrm{GHz}$ CRATES and $4.9 \mathrm{GHz}$ PMN flux densities in the spectrum of J0324-2918 (Fig. 17). Regardless of which set of points are considered, it is clear from the $148 \mathrm{MHz}$ TGSS flux density that J0324-2918 is a peaked-spectrum source. The spectral turnover would be at $\sim 1.4 \mathrm{GHz}$ or $7 \mathrm{GHz}$ (depending on which observations are considered).

There are two possible explanations for the discrepancy in flux densities between these observations. First, the AT20G values are peak brightnesses, rather than integrated flux densities. Second, the AT20G observations have a resolution between $\sim 30$ and $\sim 2 \operatorname{arcsec}$ (Murphy et al. 2010 ), the $4.9 \mathrm{GHz}$ PMN observations have a resolution of 4.2 arcmin and we could not determine the resolution of the $8.4 \mathrm{GHz}$ CRATES observations. Resolution effects could consequently have produced the difference in flux densities. The second possibility is that the difference is due to variability. J0324-2918 is a VLBI calibrator (Petrov et al. 2006) and in CFC2016 we concluded that its VLBI emission is Doppler-boosted, which strengthens the argument that it is variable.

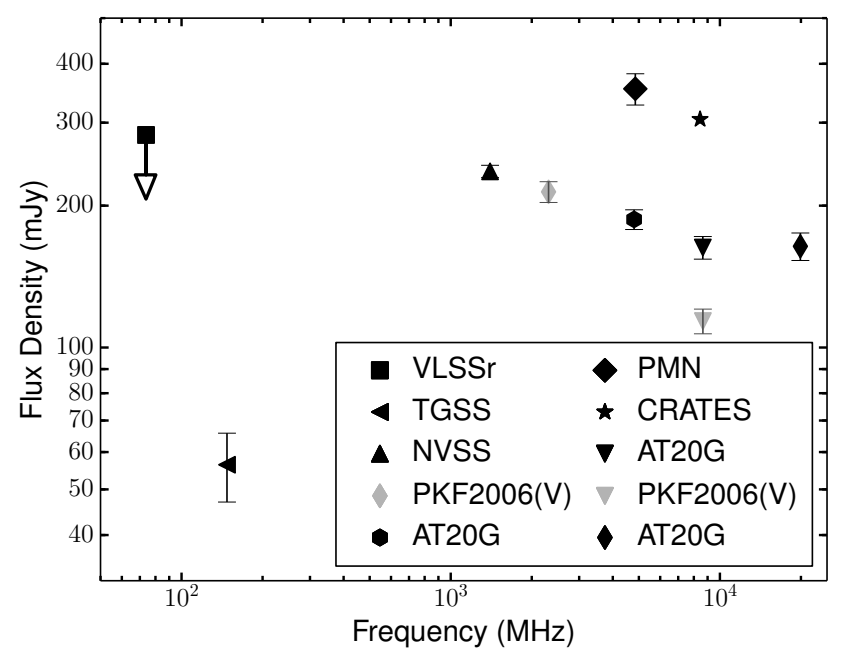

Figure 17. The radio spectrum of J0324-2918.

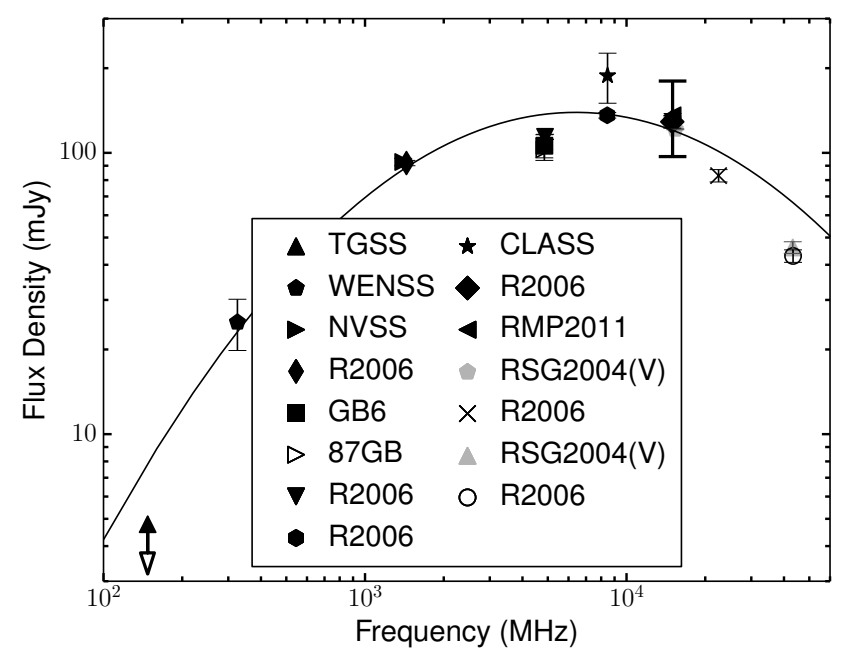

Figure 18. The radio spectrum of J0906+6930. The solid line shows the fitted log parabola. The range of flux density values between which RMP2011 observed $15 \mathrm{GHz}$ variability is indicated by the thick uncertainty bar.

\subsection{2 $50906+6930$}

The spectrum of J0906+6930 (Fig. 18) shows a clear spectral turnover. RMP2011 observed J0906+6930 55 times at $15 \mathrm{GHz}$ between 2009 March 19 and 2009 December 29. During this time they observed the flux density to vary between 97 and $180 \mathrm{mJy}$. As the source is variable, the value in Fig. 18 is the intrinsic mean $15 \mathrm{GHz}$ flux density (136 $\pm 2 \mathrm{mJy}$ ) calculated by RMP2011. Fitting the spectrum, we find a turnover frequency of $6.4 \pm 0.8 \mathrm{GHz}$. Since J0906+6930 is at $z=5.47$, this translates to a rest-frame turnover frequency of $41.4 \pm 5.2 \mathrm{GHz}$. Considering that J0906+6930 is variable and that the fitted function does not fit the $148 \mathrm{MHz}$ TGSS upper limit and the flux densities above $20 \mathrm{GHz}$ very well, the uncertainty on the turnover frequency is likely underestimated. 


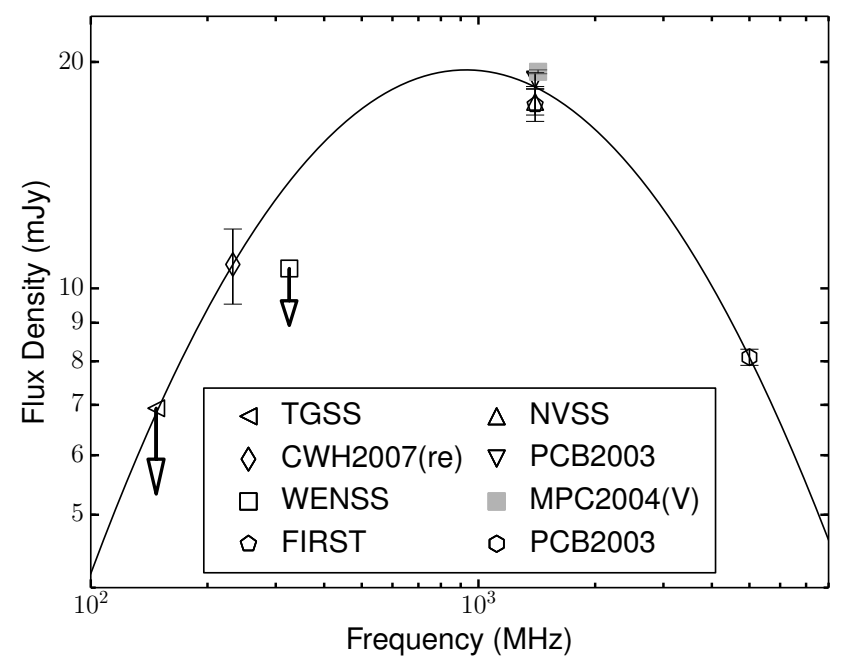

Figure 19. The radio spectrum of J0913+5919. The solid line shows the fitted log parabola.

\section{$4.3 .3 \quad J 0913+5919$}

CWH2007 found a $233 \mathrm{MHz}$ flux density of $30 \pm 3 \mathrm{mJy}$ for J0913+5919 which is incompatible with the 148 and $325 \mathrm{MHz}$ upper limits of 6.9 and $10.6 \mathrm{mJy}$ from TGSS and WENSS, respectively, in the spectrum of J0913+5919 (Fig. 19). To check this apparent discrepancy, we reprocessed the same data used by CWH2007. The raw visibility data, available from the GMRT archive under project code 04CCA01, consist of three observing sessions (2003 September 15 to 17) with a total of 11.4 hours on source. It was recorded over $4 \mathrm{MHz}$ of bandwidth centered on $232.5 \mathrm{MHz}$ and used the calibrator 3C48. We extracted the flux densities in the same way as described in Section 2. This yielded an image with a local rms noise level of $0.36 \mathrm{mJy}$ beam $^{-1}$ at a resolution of $16.4 \times 10.5 \mathrm{arcsec}$, with a beam position angle of $3^{\circ}$.

The integrated flux density of J0913+5919 in the reprocessed image is $10.7 \pm 1.2 \mathrm{mJy}$, which is a factor $\sim 3$ lower than what was found by CWH2007. The new value is compatible with the TGSS and WENSS upper limits. In the initial (preliminary) image created by our pipeline, there were strong image-plane ripples in the central region near the source. This was a rather common feature in older (hardware-correlator-based) GMRT data, and is likely the result of baseline-based errors. It is not straightforward to suppress, and might have affected the flux density measurement in CWH2007. The SPAM pipeline has dedicated image-based flagging routines to excise the visibility data causing these artefacts, yielding ripple-free images. We will therefore continue using the new flux density which is labeled as CWH2007(re) in Fig. 19.

Fitting a log parabola to the spectrum gives $\nu_{\mathrm{o}}=928 \pm$ $89 \mathrm{MHz}$, which translates to a rest-frame turnover frequency of $5670 \pm 544 \mathrm{MHz}$. We note that due to the lack of spectral coverage, the uncertainty on the turnover frequency is likely underestimated.

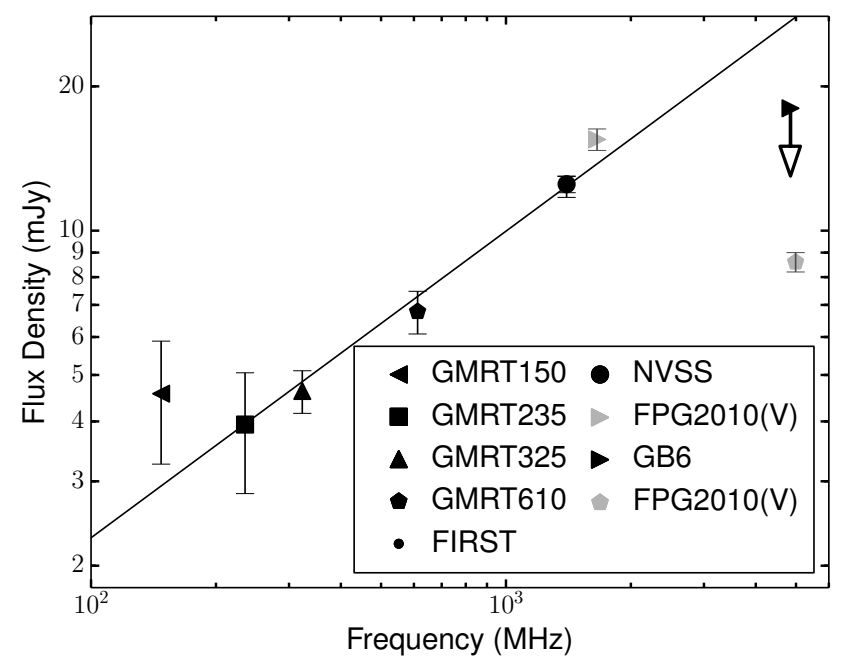

Figure 20. The radio spectrum of J1146+4037. A power law fit to the spectrum is shown as a solid line.

\subsubsection{J1146+4037}

If we were to fit a power law to the spectrum of J1146+4037 (excluding the upper limits and VLBI observations), it would give a spectral index of $\alpha=0.64 \pm 0.05$ (see Fig. 20). However, the predicted flux density at $4850 \mathrm{MHz}$ would then be $\sim 27 \mathrm{mJy}$, which is well above the $4.9 \mathrm{GHz}$ GB6 upper limit of $18 \mathrm{mJy}$. It is therefore most likely that the spectrum flattens towards higher frequencies, and considering that the spectral index between the 1.7 and $5 \mathrm{GHz}$ of the FPG2010(V) VLBI points is $-0.53 \pm 0.06$ (Coppejans et al. 2016), it appears to turn over. While care should be taken when comparing non-VLBI and VLBI spectral indices, we believe it is justified in this case, as the $1.4 \mathrm{GHz}$ FIRST and 1.6 GHz FPG2010(V) flux densities are comparable $(12.4 \pm 0.6$ and $15.5 \pm 0.8 \mathrm{mJy}$, respectively). Crucially, the GB6 upper limit also indicates a turnover. We therefore conclude that J1146+4037 likely has a spectral turnover around $1.4 \mathrm{GHz}$ and we classify it as a peakedspectrum source.

\subsubsection{J1235-0003}

It is clear that J1235-0003 has a peaked spectrum (Fig. 21). However, due to a lack of spectral coverage, we can not constrain the location of the spectral peak.

\subsubsection{J1242+5422}

Fitting a power law between the $1.4 \mathrm{GHz}$ FIRST, $1.4 \mathrm{GHz}$ NVSS and $612 \mathrm{MHz}$ GMRT610 flux densities in the spectrum of J1242+5422 (Fig. 22) gives $\alpha=-0.49 \pm 0.05$. Fitting a power law (the dashed line in Fig. 22) between all of the non-VLBI flux densities excluding FIRST and NVSS, gives $\alpha=0.12 \pm 0.06$. J1242 +5422 therefore has a positive spectral index below $\sim 610 \mathrm{MHz}$ and a negative spectral index above $\sim 610 \mathrm{MHz}$, and is therefore a peaked-spectrum source. This conclusion is supported by the $4.9 \mathrm{GHz}$ GB6 upper limit. 


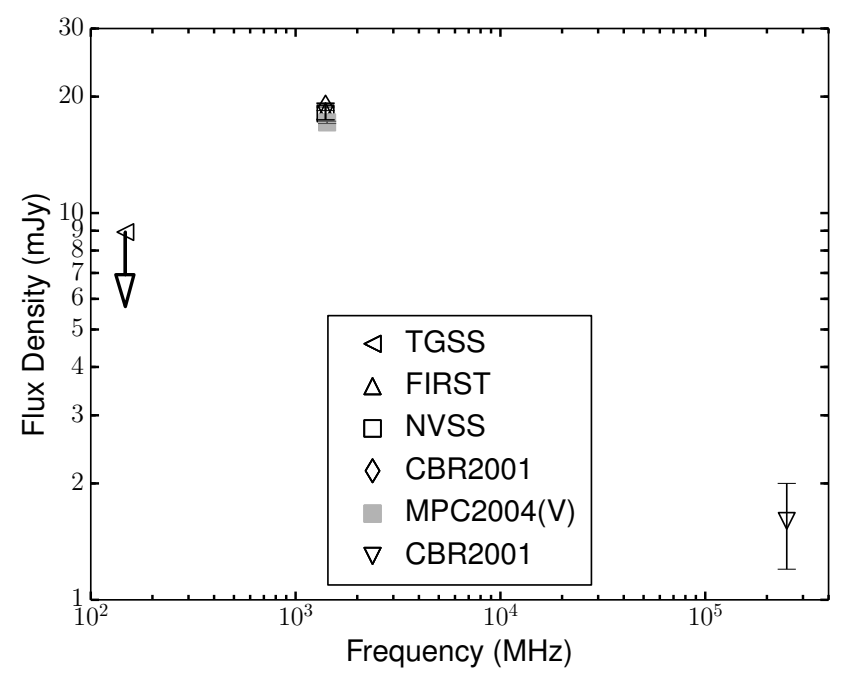

Figure 21. The radio spectrum of J1235-0003.

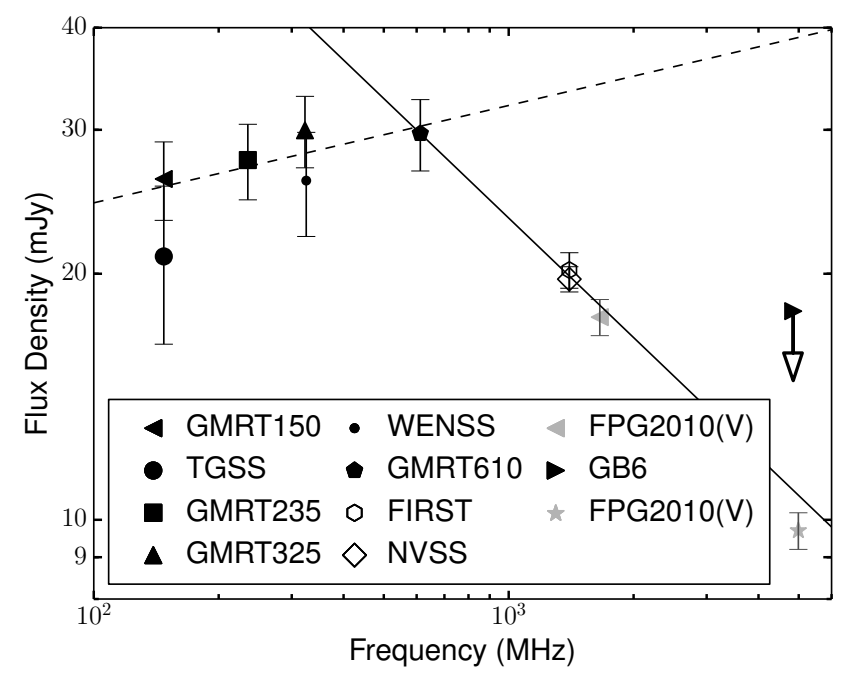

Figure 22. The radio spectrum of $\mathrm{J} 1242+5422$. The solid line is fitted between the $612 \mathrm{MHz}$ GMRT610, FIRST $(1.4 \mathrm{GHz})$ and NVSS $(1.4 \mathrm{GHz})$ flux densities, while the dashed line is fitted between all of the non-VLBI flux densities excluding FIRST and NVSS.

\section{$4.3 .7 \quad J 1606+3124$}

Matching the VLBI position of J1606+3124 to FIRST, we find that there are five sources within three arcminutes, with the nearest neighbour at a distance of 70 arcsec. In the survey catalogue these sources are indicated to have side lobe probabilities between 0.272 and 0.439 (Helfand et al. 2015). The VLA beam pattern is also clearly visible in the image, and all five neighbouring sources lie on this beam pattern ${ }^{6}$. As the $1.4 \mathrm{GHz}$ NVSS and $325 \mathrm{MHz}$ WENSS images show that the nearest neighbour is at a distance of 232 arcsec from J1606+3124 and based on the probabilities of the sources

${ }^{6}$ http://third.ucllnl.org/cgi-bin/firstcutout being side lobes, we conclude that the five neighbouring sources in the $1.4 \mathrm{GHz}$ FIRST image are all image artefacts. We matched J1606+3124 to sources in the 0.96, 2.3, 3.9, 7.7, 11.2 and $21.65 \mathrm{GHz}$ catalogues of Kovalev et al. (1999) and the 1.1, 2.3, 4.8, 7.7, 11.2 and $21.7 \mathrm{GHz}$ catalogues of Mingaliev et al. (2012). However, since these observations were taken with the RATAN-600 telescope, the resolution of all of the observations is lower than the distance to the nearest neighboring source. The flux density of the nearby sources will therefore blend with that of J1606+3124 and we discarded the matches.

The spectrum of J1606+3124 is shown in Fig. 23. RMP2011 observed J1606+3124 98 times at $15 \mathrm{GHz}$ between 2008 January 1 and 2009 December 28 with the $40 \mathrm{~m}$ telescope at the Owens Valley Radio Observatory. From this they concluded that $\mathrm{J} 1606+3124$ is not variable. While we discarded the matches to Mingaliev et al. (2012), we note that the authors did observe J1606+3124 six times with the RATAN-600 telescope between 2006 July and 2010 May at $21.7,11.2,7.7,4.8$ and $2.3 \mathrm{GHz}$, and five times at $1 \mathrm{GHz}$ over the same period. These observations also indicate that $\mathrm{J} 1606+3124$ is not variable at these frequencies. The average $15 \mathrm{GHz}$ flux density of RMP2011 at each frequency are plotted in Fig. 23. In OP1987 the authors give the $90 \mathrm{GHz}$ flux density as $10 \pm 150 \mathrm{mJy}$. Since the uncertainty is nonphysically large we omitted it in Fig. 23. We do however note that it is possible that the uncertainty is correct and the value itself is wrong.

It has been known for some time that J1606+3124 has a peaked spectrum (e.g. Spoelstra et al. 1985), with De Vries et al. (1997) and Mingaliev et al. (2013) reporting peak frequencies of 1.5 and $3.5 \mathrm{GHz}$, respectively. Fitting a $\log$ parabola to the spectrum, we found $\nu_{\mathrm{o}}=2581 \pm$ $536 \mathrm{MHz}$. Taking into account the redshift of J1606+3124, our observed turnover frequency translates to a rest-frame turnover frequency of $14.4 \pm 3.0 \mathrm{GHz}$. We finally note that in the $4.8 \mathrm{GHz}$ HTT2007(V) and in the 2.2 and $8.3 \mathrm{GHz}$ BGP2002(V) VLBI observations, J1606+3124 has a Compact Symmetric Object (CSO) structure. CSOs are characterised by unbeamed emission from their steep-spectrum radio lobes on either side of a central position, and have sizes smaller than their host galaxy (Fanti et al. 1995; Fanti 2009).

\subsubsection{J1659+2101}

The $148 \mathrm{MHz}$ TGSS and $147 \mathrm{MHz}$ GMRT150 flux densities in the spectrum of J1659+2101 (Fig. 24) are 27.6 \pm 5.7 and $48.2 \pm 5.4 \mathrm{mJy}$, respectively. This translates to a difference of $1.9 \sigma$ or 75 per cent in flux density. Visual inspection of the images did not reveal an explanation for the offset. To try find an explanation, we matched the sources in the $147 \mathrm{MHz}$ GMRT150 image to those in TGSS using a 10 arcsec search radius. We found 22 matches within a square of $1 \times 1 \mathrm{deg}$ centred on J1659+2101. For each of these sources, we calculated the ratio between the $147 \mathrm{MHz}$ GMRT150 and the $148 \mathrm{MHz}$ TGSS flux densities: The median of all of the ratios was 0.95 , and the average was 1.02 . The discrepancy can consequently not be attributed to a systematic flux density offset between the catalogues. Another possible explanation for the difference could be that J1659+2101 is variable. This is contradicted, but not ruled out, by the $1.4 \mathrm{GHz}$ FIRST 


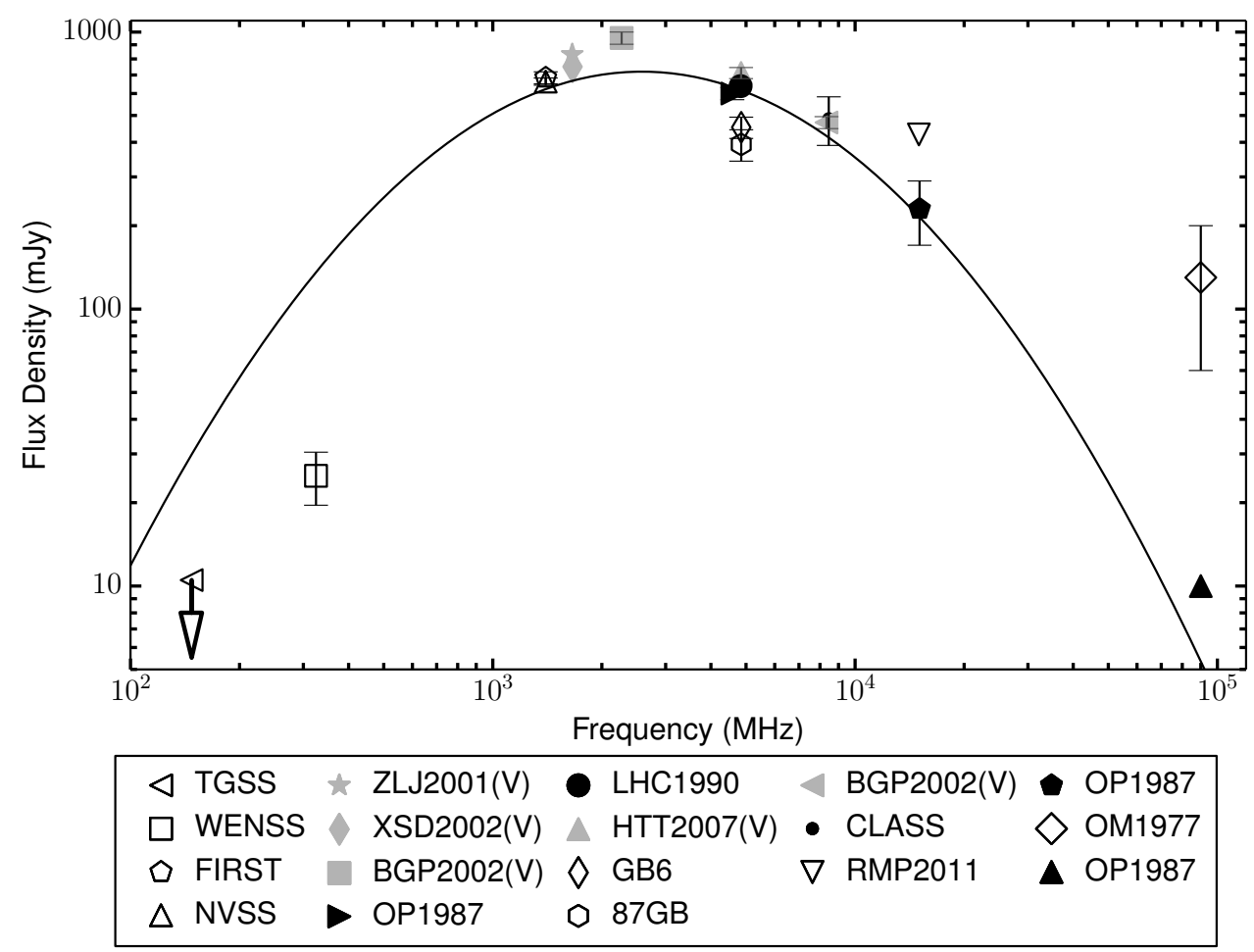

Figure 23. The radio spectrum of J1606+3124. The solid line shows the fitted log parabola.

and NVSS flux densities that are within 2 per cent of each other despite the epochs when FIRST and NVSS observed J1659+2101 differing by about 3.4 years (Ofek \& Frail 2011; Helfand et al. 2015). Resolution effects also cannot explain the difference, as the resolutions of the surveys are similar $(25 \times 25$ arcsec and $23 \times 16$ arcsec, respectively). We can therefore not explain the difference between the TGSS and GMRT150 flux densities.

Fitting a power law to the spectrum, and excluding the TGSS and GMRT150 flux densities, gives $\alpha=-0.40 \pm 0.05$. Repeating the fit using only the GMRT150 and $235 \mathrm{MHz}$ GMRT235 values give $\alpha=0.27 \pm 0.33$, while fitting only the TGSS and GMRT235 values gives $\alpha=1.47 \pm 0.49$. It is therefore clear that irrespective of whether the TGSS or the GMRT150 flux densities are correct, at the very least the spectrum flattens, and it likely turns over around $235 \mathrm{MHz}$. We therefore classify J1659+2101 as having a peaked spectrum.

\subsubsection{J2102+6015}

The spectrum of J2102+6015 (Fig. 25) shows a clear turnover. Fitting the spectrum with a log parabola gives $\nu_{\mathrm{o}}=1031 \pm 51 \mathrm{MHz}$. This corresponds to a rest-frame turnover frequency of $5753 \pm 283 \mathrm{MHz}$.

\section{$4.3 .10 \quad$ J2228+0110}

Despite J2228+0110 only being detected in the $1.4 \mathrm{GHz}$ STRIPE82 survey, the $3 \mathrm{GHz}$ CNSS and $148 \mathrm{MHz}$ TGSS upper limits show that its spectrum (Fig. 26) peaks below $1.4 \mathrm{GHz}$.

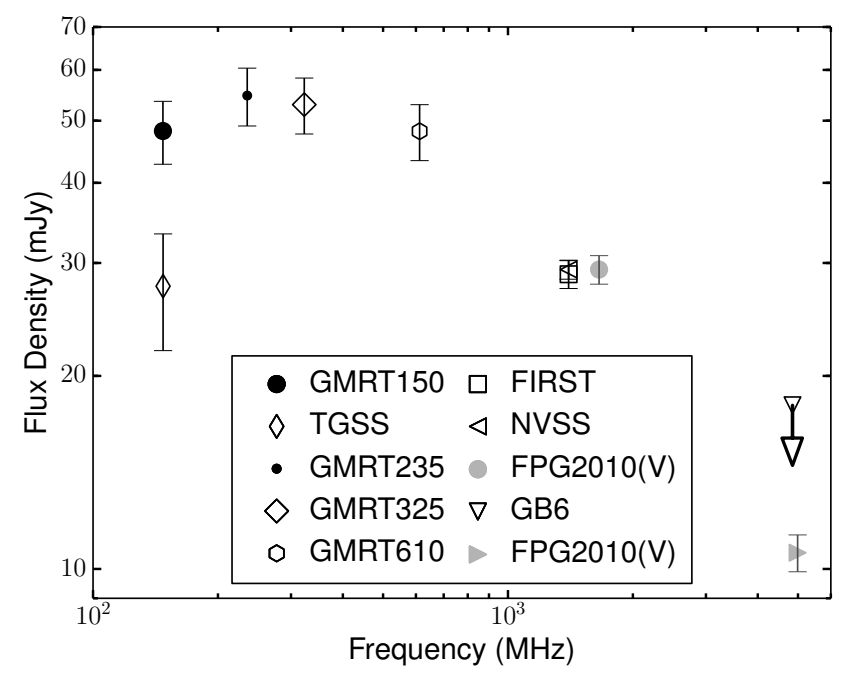

Figure 24. The radio spectrum of J1659+2101.

\subsection{Unusual and unclassified spectra}

The last class contains the six sources that cannot be classified into one of the three previous classes, and those that (due to a lack of spectral coverage) could have spectra that fall into more than one of the classes.

\section{$4 \cdot 4 \cdot 1 \quad J 1013+2811$}

Assuming that the spectrum of J1013+2811 (Fig. 27) can be fitted with a single power law, and using only the $1.4 \mathrm{GHz}$ 


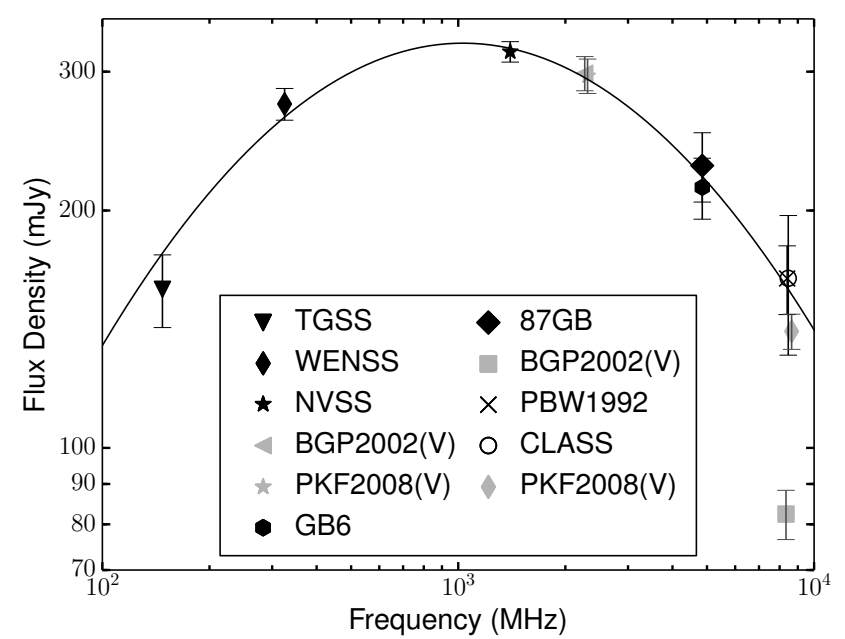

Figure 25. The radio spectrum of J2102+6015. The solid line shows the fitted log parabola.

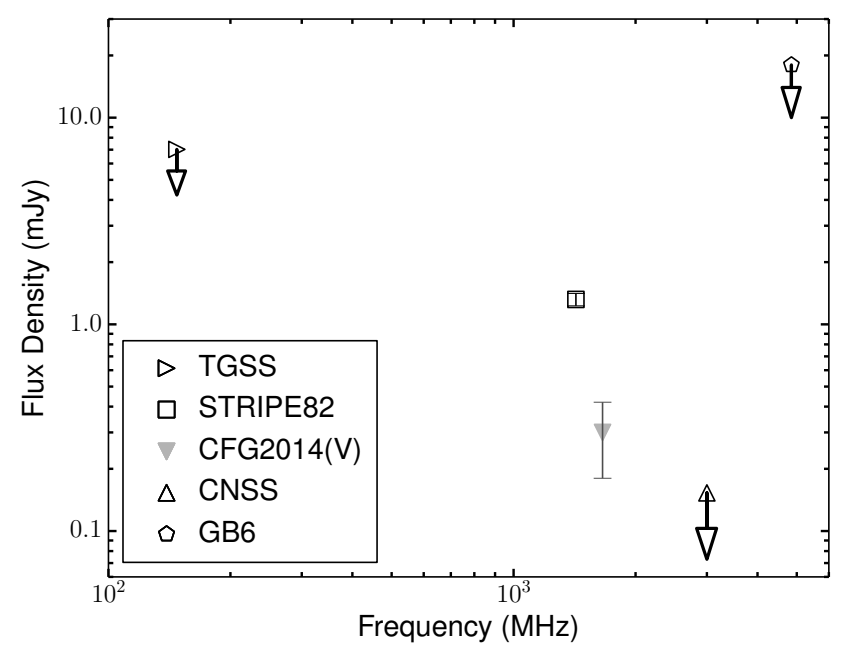

Figure 26. The radio spectrum of J2228+0110.

FIRST flux density and the $4.9 \mathrm{GHz}$ GB6 upper limit, produces a spectral index $\alpha<0.18$. Similarly, a fit using only the FIRST flux density and the $148 \mathrm{MHz}$ TGSS upper limit, produces a spectral index greater than zero. Based on these limits, J1013+2811 can either have a flat or a peaked spectrum.

\subsubsection{J1205-0742}

The spectrum of J1205-0742 (Fig. 28) is concave, with evidence of variability at $1.4 \mathrm{GHz}$. Using its spectral index between 1.4 and $350 \mathrm{GHz}$, morphology, brightness temperature and linear size, MCP2005(V) showed that the radio emission from J1205-0742 is from a nuclear starburst, and that the source does not have a radio-loud AGN. This explains why J1205-0742 has a concave spectrum. At $\nu_{\mathrm{o}}<100 \mathrm{GHz}$, the negative spectral index is caused by starburst-driven radio synchrotron emission, while at $\nu_{\mathrm{o}} \gtrsim 100 \mathrm{GHz} \simeq \nu_{\mathrm{r}} \gtrsim$ $570 \mathrm{GHz}$, the increase in flux density is the result of thermal

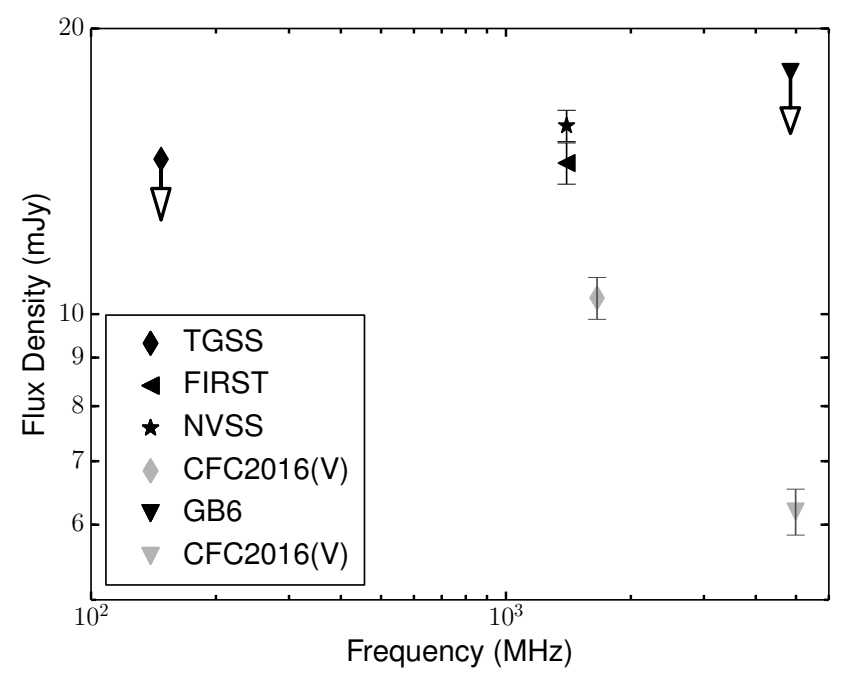

Figure 27. The radio spectrum of $\mathrm{J} 1013+2811$.

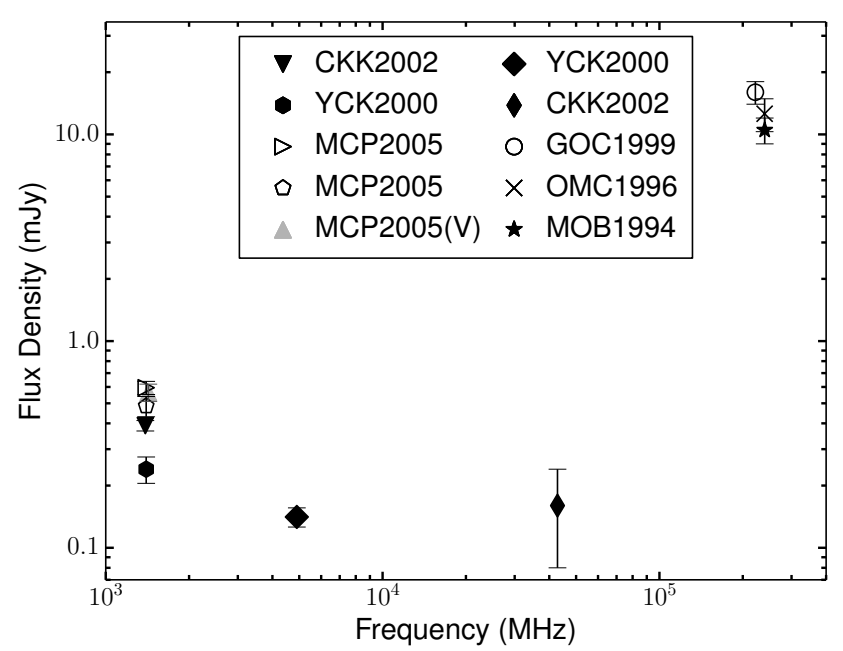

Figure 28. The radio spectrum of J1205-0742.

dust emission (e.g. McMahon et al. 1994; Yun et al. 2000; Momjian et al. 2005; Planck Collaboration et al. 2011).

\section{$4.4 .3 \quad J 1311+2227$}

Assuming that the spectrum of J1311+2227 (Fig. 29) can be fitted with a single power law, and using the $1.4 \mathrm{GHz}$ FIRST flux density and the $148 \mathrm{MHz}$ TGSS and $4.9 \mathrm{GHz}$ GB6 upper limits, the spectral index is $-0.19<\alpha<0.84$. J1311+2227 can therefore either have a flat, inverted or peaked spectrum.

\section{$4.4 .4 \quad J 1454+1109$}

Based on the VLBI flux densities being higher than the non-VLBI flux densities in the spectrum of J1454+1109 (Fig. 30), and the $4.9 \mathrm{GHz}$ GB6 upper limit and the $1.4 \mathrm{GHz}$ FIRST flux density being higher than the $1.4 \mathrm{GHz}$ NVSS flux density, we conclude that $\mathrm{J} 1454+1109$ is variable. In 


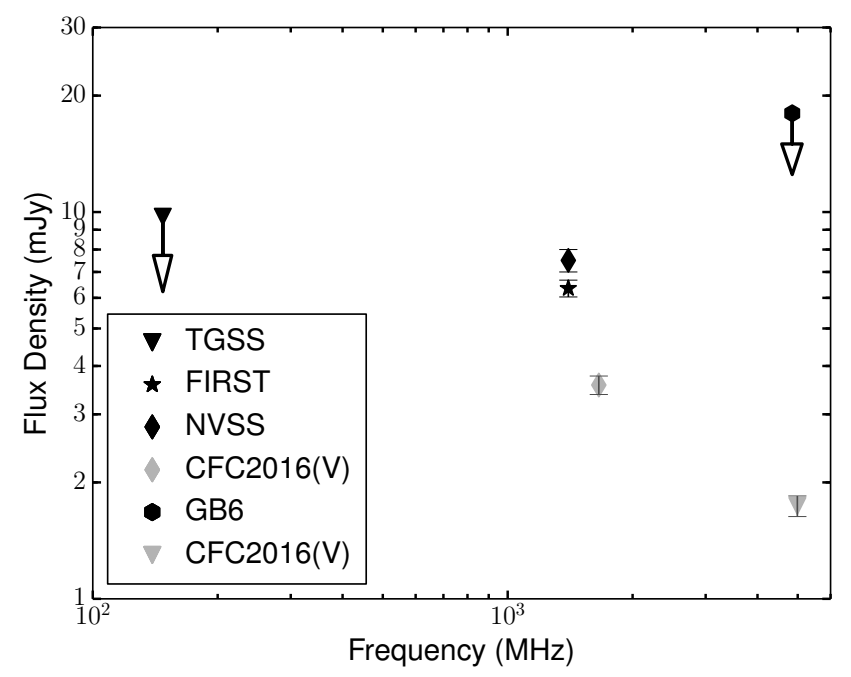

Figure 29. The radio spectrum of $\mathrm{J} 1311+2227$.

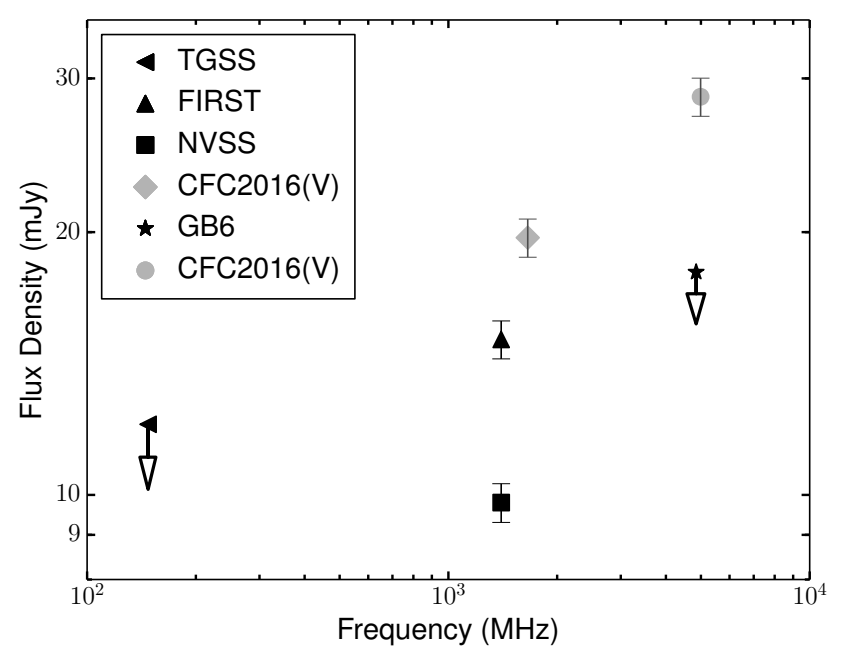

Figure 30. The radio spectrum of J1454+1109.

addition, due to a lack of spectral coverage, we cannot constrain the spectrum. However, based on its variability, and the fact that the VLBI emission is Doppler-boosted (Coppejans et al. 2016), J1454+1109 is likely a blazar with a flat time-averaged spectrum.

\subsubsection{J1611+0844}

Assuming that the spectrum of J1611+0844 (Fig. 31) can be fitted with a single power law, and using the $1.4 \mathrm{GHz}$ FIRST flux density and the $148 \mathrm{MHz}$ TGSS and $4.9 \mathrm{GHz}$ GB6 upper limits, $-0.06<\alpha<0.57$. The time-averaged spectrum can therefore be either inverted, flat or peaked. Since the VLBI flux densities are higher than the non-VLBI flux densities, it is likely that J1611+0844 is variable. However, since the epochs when FIRST and NVSS $(1.4, \mathrm{GHz})$ observed J1611+0844 differ by about 3.6 years (Ofek \& Frail 2011; Helfand et al. 2015), if J1611+0844 is variable it means

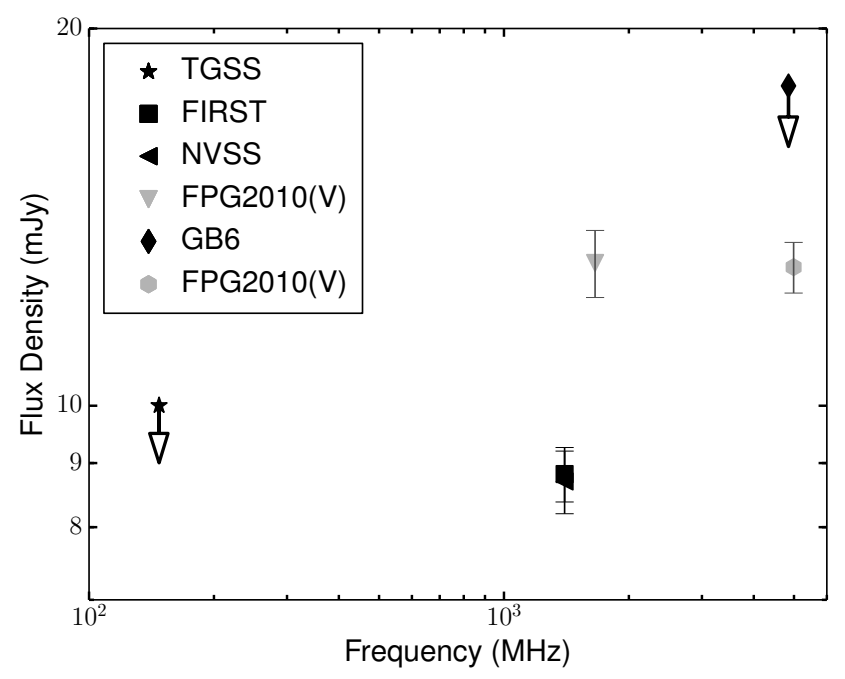

Figure 31. The radio spectrum of J1611+0844.

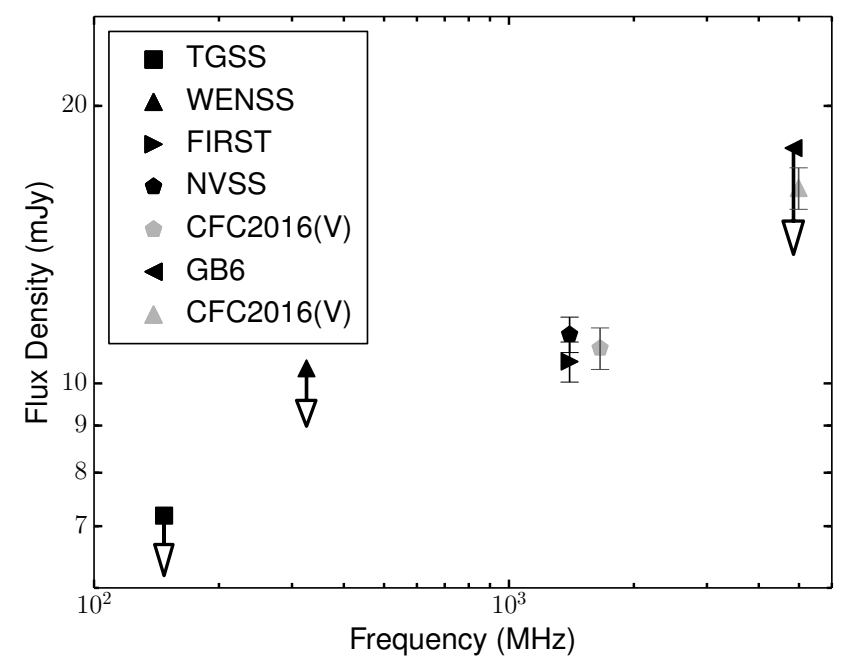

Figure 32. The radio spectrum of J1720+3104

that the FIRST and NVSS observations were serendipitously done on two epochs when J1611+0844 happened to have the same flux density.

\section{$4.4 .6 \quad J 1720+3104$}

Assuming that the spectrum of J1720+3104 (Fig. 32) can be fitted with a single power law, and using the $1.4 \mathrm{GHz}$ FIRST flux density, and the $148 \mathrm{MHz}$ TGSS (which is more constraining than $325 \mathrm{MHz}$ WENSS value) and $4.9 \mathrm{GHz}$ GB6 upper limits, $0.17<\alpha<0.43$. This is consistent with the spectral index of $\alpha=0.36 \pm 0.07$ measured between the 1.7 and $5 \mathrm{GHz}$ CFC2016(V) VLBI flux densities. J1720+3104 can therefore have either a flat or a peaked spectrum. 
Table 7. Spectral classification summary

\begin{tabular}{ccccc}
\hline \multirow{2}{*}{$\begin{array}{c}\text { Spectral } \\
\text { classification }\end{array}$} & $\begin{array}{c}\text { Full sample } \\
\text { Number of } \\
\text { sources }\end{array}$ & $\begin{array}{c}\text { \% of } \\
\text { sources }\end{array}$ & $\begin{array}{c}\text { Number of } \\
\text { sources }\end{array}$ & $\begin{array}{c}\text { Unbiased of } \\
\text { sources }\end{array}$ \\
\hline Inverted & $0_{-0}^{+3}$ & $0_{-0}^{+10}$ & $0_{-0}^{+3}$ & $0_{-0}^{+14}$ \\
Flat & $6_{-1}^{+5}$ & $21_{-3}^{+17}$ & $5_{-1}^{+5}$ & $23_{-5}^{+23}$ \\
Steep & $8_{-3}^{+1}$ & $28_{-10}^{+3}$ & $7_{-2}^{+1}$ & $32_{-9}^{+5}$ \\
USS & $0_{-0}^{+2}$ & $0_{-0}^{+7}$ & $0_{-0}^{+1}$ & $0_{-0}^{+5}$ \\
Peaked & $10_{-1}^{+4}$ & $34_{-3}^{+14}$ & $6_{-1}^{+4}$ & $27_{-5}^{+18}$ \\
Concave & $1_{-0}^{+0}$ & $3_{-0}^{+0}$ & $0_{-0}^{+0}$ & $0_{-0}^{+0}$ \\
\hline
\end{tabular}

Notes: ${ }^{a}$ The format $b_{-d}^{+c}$ should be interpreted as follows: There are $b$ sources in the given spectral class, and an additional $c$ sources that are not in the class but could be. Of the $b$ sources, $d$ are in the class but could have a different spectral classification within the errors on their spectral indices.

\section{DISCUSSION}

In Table 7, the number and the percentage of sources in each spectral class are given for the full sample and unbiased subsample (which is described later in this section; see the table caption for a description of the nomenclature used). This table was compiled from the classifications in Table 4 in the following way: (1) if a source is classified as e.g. 'Flat' in Table 4 , then the number of flat-spectrum sources is increased by one; (2) if a source is classified as 'flat (steep)', then the number of flat-spectrum sources is increased by one, the lower uncertainty on the number of flat-spectrum sources is decreased by one, and the upper uncertainty on the number of steep-spectrum sources is increased by one; (3) if a source is classified as 'flat or peaked', the upper uncertainty on the number of flat-spectrum and peaked-spectrum sources are both increased by one. Finally, the percentage of sources in each class of the full sample were calculated using a total number of 29 sources, since the spectrum of J1454+1109 is completely unconstrained (Section 4.4.4). We also point out again, that as discussed in Section 1, in all of the sources except J1205-0742 (which has a concave spectrum), the radio emission is caused by AGN activity. In J1205-0742 the radio emission is caused by star formation.

The primary selection effects in our sample of sources are that all of the sources have spectroscopic redshifts and were selected for follow-up high-resolution VLBI observations. In general the latter involves a flux density lower limit and the sources being compact on arcsec scales in previous (e.g. FIRST) observations. In addition, some authors selected sources for VLBI observations because of the shape of their radio spectra. Since this can bias the values of the full sample in Table 7, we created a unbiased sub-sample of sources that were not selected for VLBI observation with a spectral bias. To do this we checked how each of the sources was selected for VLBI observation the first time that they were observed. If a source was selected for VLBI observations with a spectral bias it was not included in the unbiased subsample. This resulted in the following seven sources not being in the unbiased sub-sample: J0311+0507, J0324-2918, $\mathrm{J} 0906+6930, \mathrm{~J} 1026+2542$, J1205-0742, J1606+3124 and J2102+6015. In columns 4 and 5 of Table 7 we re-calculated the values in columns 2 and 3 for our unbiased sub-sample. The percentage of sources in each class of the unbiased sub- sample was calculated using a total number of 22 sources, since the spectrum of J1454+1109 is completely unconstrained (Section 4.4.4).

In Table 7 , the fact that we did not find a single USS sources is striking considering that the USS technique is specifically used to search for high-redshift sources. All of the VLBI observations of the sources were carried out above $1.4 \mathrm{GHz}$ (Table A1), where the flux densities of the USS sources are rapidly decreasing (Section 4.2). The lack of USS sources could therefore be the result of sources typically only being considered for VLBI observation if, in previous nonVLBI observations, they have flux densities above a certain minimum.

To attempt to test if this is the case, we downloaded the 12th data release of the Sloan Digital Sky Survey quasar catalog (Pâris et al. 2017) and removed all sources with SDSS pipeline redshifts smaller than 4.5. Of the remaining 1054 sources, 16 are VLBI sources discussed in this paper. Using a search radius of 5 arcsec, we matched all the sources in FIRST $(1.4 \mathrm{GHz})$ to the list of $z>4.5$ SDSS sources and the TGSS catalogue $(148 \mathrm{MHz})$. From this we found 22 sources which have both FIRST and TGSS flux densities, and of these, six are in this paper. Removing these six sources and calculating two-point spectral indices between FIRST and TGSS for the remaining sources, we found one USS source and one source that could be a USS source within its uncertainties. We do, however, note that since the FIRST and TGSS typical detection thresholds are 1 and $35 \mathrm{mJy}$, respectively, only USS sources with FIRST flux densities above 4 mJy will be detected in TGSS. Only $6_{-0}^{+6}$ per cent of the FIRST-TGSS sources are USS sources. This is in agreement with the percentages of USS sources in Table 7. As the fraction of USS sources in these three samples are consistent, it is likely that the requirements for VLBI follow-up observations do not produce a bias against USS sources.

The largest group of sources in the full sample, and the second largest group of sources in the unbiased subsample, are the peaked-spectrum sources. These sources are believed to be young AGN (e.g. O'Dea 1998; Conway 2002; Murgia et al. 2002; Murgia 2003; Orienti 2016) and make up more than a quarter of the sources in our unbiased sub-sample. Of the 10 peaked-spectrum sources in the sample, sufficient spectral coverage is available to determine the observed turnover frequency of seven of them to within $\sim 1 \mathrm{GHz}$ (Section 4). For two of these sources (J0913+5919 and J1659+2102), the observed spectral turnover lies below $1 \mathrm{GHz}$, and for three more sources $(\mathrm{J} 1242+5422, \mathrm{~J} 2102+6015$ and J2228+0110) the observed turnover could lie below $1 \mathrm{GHz}$ (but definitely lies below $\sim 2 \mathrm{GHz}$ ). The final two sources (J0906+6930 and J1606+3124) both have observed spectral turnovers above $\sim 3 \mathrm{GHz}$. Consequently, the peaked-spectrum sources show a wide range of observed turnover frequencies, and an even wider range of rest-frame turnover frequencies. Based on their observed turnover frequencies, the peaked-spectrum sources are MPS, GPS and HFP sources. This also shows that there are between two and four MPS sources in the unbiased sub-sample. Consequently, although there are more MPS sources than USS sources, neither of these methods would have selected more than $\sim 18$ per cent of the sources in the unbiased sub-sample. Interestingly, four of the sources (J0324-2918, J0906+6930, J1606+3124 and J2102+6015) 
that were excluded from the unbiased sub-sample were excluded because they were selected for VLBI observation based on having flat two-point spectral indices (Beasley et al. 2002; Romani et al. 2004; Petrov et al. 2006). However, all four sources actually have peaked spectra and only appeared to have flat spectra because their spectral indices were determined close to the spectral peak.

It is worth noting that the spectra of the steep, and USS, sources have to turn over at some point due to synchrotron self-absorption. In addition, assuming $z=5$, any source with a rest-frame spectral turnover below $\sim 3 \mathrm{GHz}$ will appear as a steep-spectrum source in our sample since the observed frame turnover frequency will be below $\sim 300 \mathrm{MHz}$. For six of the steep-spectrum and USS sources in the sample, the turnover has to be below an observed frequency $1 \mathrm{GHz}$, while for two of the sources it has to be below $1.4 \mathrm{GHz}$ (Section 4). In total there are $13_{-2}^{+5}$ sources that are steep, USS or peaked in the unbiased sub-sample, which translates to $59_{-9}^{+23}$ per cent of the sources in the unbiased sub-sample. It is therefore safe to say that, if the steep-spectrum sources are observed at lower frequencies $\left(\nu_{\mathrm{o}}<100 \mathrm{MHz}\right)$, more of the sources in both the sample and unbiased sub-sample would be classified as peaked-spectrum sources, and there would likely be significantly more MPS sources.

In CFC2016 we pointed out that the selection effects discussed previously likely bias the sample towards flatspectrum sources in which the radio emission is Dopplerboosted (which increases the sources' flux density). It was therefore surprising that we found that less than half of the sources could be classified as flat-spectrum radio quasars (Coppejans et al. 2016). This conclusion is supported by our new finding that $23_{-5}^{+23}$ per cent of the sources in the unbiased sub-sample have flat spectra.

\section{SUMMARY AND CONCLUSIONS}

In this paper, we presented new multi-frequency GMRT observations at $\nu<1 \mathrm{GHz}$ of eight $z>4.5$ VLBI sources. Matching these eight, and the remaining $22 z>4.5$ VLBI sources, to the literature, we constructed broad-band radio spectra of all $30 z>4.5$ VLBI sources. We then discussed and classified the spectra of each of the sources as flat, steep, peaked, unusual and unclassified. Next we looked at the properties of the sample - particularly the fraction of sources in each spectral class. There are no USS sources in the sample, which we argued is not caused by the requirements for VLBI follow-up observations producing a bias against USS sources. We also show that the USS and MPS methods would each have selected less than $\sim 5$ and $\sim 18$ per cent of the sources in the sample, respectively. This supports the argument by Pedani (2003) that the USS sources are not representative of the entire high-redshift source population. We do note that because of the small number of MPS and USS sources in the sample, larger samples are required to draw a definitive conclusion.

The spectra of the steep-spectrum sources have to turn over at some point. If these sources are observed at lower frequencies $\left(\nu_{\mathrm{o}}<100 \mathrm{MHz}\right)$, the percentage of peakedspectrum and MPS sources in the sample would likely increase significantly. This would result in even more MPS than USS sources. We also note that due to a lack of spec- tral coverage, the classification of some of the sources is uncertain. This problem can be resolved with multi-frequency observations below $2 \mathrm{GHz}$, since, for a source at $z=5$, its entire rest-frame spectrum below $12 \mathrm{GHz}$ will be shifted into observed frequencies below $2 \mathrm{GHz}$.

The most striking feature of Table 7 is that there is no single spectral class that has the majority of sources. The sources are spread roughly evenly between the flat, steep and peaked classes. In addition, in one of the sources the radio emission is related to star-forming activity. Despite several selection effects, we have to conclude that the $z>4.5 \mathrm{VLBI}$ sources (and likely also the $z>4.5$ non-VLBI sources) have diverse radio spectra. Considering that we expect the Square Kilometre Array (SKA) to be able to detect sources out to beyond redshift 10 (e.g. Falcke et al. 2004), and knowing the general importance of these sources, it is critical that methods are found with which to reliably identify complete samples of high-redshift sources based on radio data.

\section{ACKNOWLEDGEMENTS}

The authors wish to thank the referee, Heinz Andernach, and the editor for their suggestions and comments which helped to improve this paper. We thank the staff of the GMRT who made these observations possible. The GMRT is run by the National Centre for Radio Astrophysics of the Tata Institute of Fundamental Research. Scientific results from data presented in this publication are derived from the following GMRT project codes: 21_013 (PI: S. van Velzen) and 29_007 (PI: R. Coppejans). S.F., D.C., and K.É.G. thank the Hungarian National Research, Development and Innovation Office (OTKA NN110333) for their support. C.M. and H.F. are funded by the ERC Synergy Grant BlackHoleCam: Imaging the Event Horizon of Black Holes (Grant 610058). WLW acknowledges support from the UK Science and Technology Facilities Council [ST/M001008/1]. This research has made use of the NASA/IPAC Extragalactic Database (NED) which is operated by the Jet Propulsion Laboratory, California Institute of Technology, under contract with the National Aeronautics and Space Administration, the VizieR catalogue access tool, CDS, Strasbourg, France and NASA's Astrophysics Data System.

\section{REFERENCES}

Abazajian K. N., et al., 2009, ApJS, 182, 543

An T., Baan W. A., 2012, ApJ, 760, 77

Beasley A. J., Gordon D., Peck A. B., Petrov L., MacMillan D. S., Fomalont E. B., Ma C., 2002, ApJS, 141, 13

Becker R. H., Fan X., et al., 2001, AJ, 122, 2850

Begelman M. C., 1996, in Carilli C. L., Harris D. E., eds, Proc. Workshop: 'Cygnus A - Study of a Radio Galaxy'. Cambridge Univ. Press, Cambridge. p. 209

Best P. N., Kauffmann G., Heckman T. M., Brinchmann J., Charlot S., Ivezić Ž., White S. D. M., 2005, MNRAS, 362, 25

Blumenthal G., Miley G., 1979, A\&A, 80, 13

Blundell K. M., Rawlings S., Eales S. A., Taylor G. B., Bradley A. D., 1998, MNRAS, 295, 265

Blundell K. M., Rawlings S., Willott C. J., 1999, AJ, 117, 677

Braude S. I., Megn A. V., Sokolov K. P., Tkachenko A. P., Sharykin N. K., 1979, Ap\&SS, 64, 73 
Broderick J. W., Bryant J. J., Hunstead R. W., Sadler E. M., Murphy T., 2007, MNRAS, 381, 341

Bursov N. N., 1996, Bulletin of the Special Astrophysics Observatory, 40,128

Cao H.-M., Frey S., Gurvits L. I., Yang J., Hong X.-Y., Paragi Z., Deller A. T., Ivezić Ž., 2014, A\&A, 563, A111

Carilli C. L., et al., 2001, ApJ, 555, 625

Carilli C. L., et al., 2002, AJ, 123, 1838

Carilli C. L., Wang R., van Hoven M. B., Dwarakanath K., Chengalur J. N., Wyithe S., 2007, AJ, 133, 2841

Chandra P., Ray A., Bhatnagar S., 2004, ApJ, 612, 974

Ciliegi P., et al., 1999, MNRAS, 302, 222

Colla G., et al., 1972, A\&AS, 7, 1

Condon J. J., Huang Z.-P., Yin Q. F., Thuan T. X., 1991, ApJ, 378,65

Condon J. J., Cotton W. D., Greisen E. W., Yin Q. F., Perley R. A., Taylor G. B., Broderick J. J., 1998, AJ, 115, 1693

Conway J. E., 2002, New A Rev., 46, 263

Coppejans R., Cseh D., Williams W. L., van Velzen S., Falcke H., 2015, MNRAS, 450, 1477

Coppejans R., et al., 2016, MNRAS, 463, 3260

Croft S., et al., 2010, ApJ, 719, 45

Cruz M. J., Jarvis M. J., et al., 2006, MNRAS, 373, 1531

Dallacasa D., Stanghellini C., Centonza M., Fanti R., 2000, A\&A, 363,887

De Breuck C., van Breugel W., Röttgering H. J. A., Miley G., 2000, A\&AS, 143, 303

De Breuck C., Hunstead R. W., Sadler E. M., Rocca-Volmerange B., Klamer I., 2004, MNRAS, 347, 837

De Vries W. H., Barthel P. D., O’Dea C. P., 1997, A\&A, 321, 105

De Vries W. H., O'Dea C. P., Barthel P. D., 2002, New A Rev., 46, 163

Douglas J. N., Bash F. N., Bozyan F. A., Torrence G. W., Wolfe C., 1996, AJ, 111, 1945

Fabian A. C., 2012, ARA\&A, 50, 455

Falcke H., Körding E., Nagar N. M., 2004, New A Rev., 48, 1157

Fanti C., 2009, Astronomische Nachrichten, 330, 120

Fanti C., Fanti R., Dallacasa D., Schilizzi R. T., Spencer R. E., Stanghellini C., 1995, A\&A, 302, 317

Ficarra A., Grueff G., Tomassetti G., 1985, A\&AS, 59, 255

Frey S., Mosoni L., Paragi Z., Gurvits L. I., 2003, MNRAS, 343, L20

Frey S., Paragi Z., Mosoni L., Gurvits L. I., 2005, A\&A, 436, L13

Frey S., Gurvits L. I., Paragi Z., É. Gabányi K., 2008, A\&A, 484, L39

Frey S., Paragi Z., Gurvits L. I., Cseh D., Gabányi K. É., 2010, A\&A, 524, A83

Frey S., Paragi Z., Gurvits L. I., Gabányi K. É., Cseh D., 2011, A\&A, 531, L5

Frey S., Fogasy J. O., Paragi Z., Gurvits L. I., 2013, MNRAS, 431, 1314

Frey S., Paragi Z., Fogasy J. O., Gurvits L. I., 2015, MNRAS, 446, 2921

Gabányi K. É., Cseh D., Frey S., Paragi Z., Gurvits L. I., An T., Zhang Y. K., 2015, MNRAS, 450, L57

Goss W. M., Parijskij Y. N., Soboleva N. S., Temirova A. V., Vitkovskij V. V., Zhelenkova O. P., Naugol'naya M. N., 1992, AZh, 69, 673

Gower J. F. R., Scott P. F., Wills D., 1967, MmRAS, 71, 49

Gregory P. C., Condon J. J., 1991, ApJS, 75, 1011

Gregory P. C., Scott W. K., Douglas K., Condon J. J., 1996, ApJS, 103, 427

Guilloteau S., Omont A., Cox P., McMahon R. G., Petitjean P., 1999, A\&A, 349, 363

Healey S. E., Romani R. W., Taylor G. B., Sadler E. M., Ricci R., Murphy T., Ulvestad J. S., Winn J. N., 2007, ApJS, 171, 61

Helfand D. J., White R. L., Becker R. H., 2015, ApJ, 801, 26
Helmboldt J. F., et al., 2007, ApJ, 658, 203

Hodge J. A., Becker R. H., White R. L., Richards G. T., Zeimann G. R., 2011, AJ, 142, 3

Intema H. T., Jagannathan P., Mooley K. P., Frail D. A., 2016, preprint, (arXiv:1603.04368)

Jarvis M. J., Rawlings S., et al., 2001, MNRAS, 326, 1563

Jarvis M. J., Teimourian H., Simpson C., Smith D. J. B., Rawlings S., Bonfield D., 2009, MNRAS, 398, L83

Jiang L., et al., 2014, ApJS, 213, 12

Ker L. M., Best P. N., Rigby E. E., Röttgering H. J. A., Gendre M. A., 2012, MNRAS, 420, 2644

Kewley L. J., Heisler C. A., Dopita M. A., Sutherland R., Norris R. P., Reynolds J., Lumsden S., 2000, ApJ, 530, 704

Klamer I. J., Ekers R. D., Bryant J. J., Hunstead R. W., Sadler E. M., De Breuck C., 2006, MNRAS, 371, 852

Kopylov A. I., Goss W. M., Parĭ̈skï Y. N., Soboleva N. S., Verkhodanov O. V., Temirova A. V., Zhelenkova O. P., 2006, Astronomy Letters, 32, 433

Kovalev Y. Y., Nizhelsky N. A., Kovalev Y. A., Berlin A. B., Zhekanis G. V., Mingaliev M. G., Bogdantsov A. V., 1999, A\&AS, 139, 545

Krawczynski H., Treister E., 2013, Frontiers of Physics, 8, 609

Laing R. A., Peacock J. A., 1980, MNRAS, 190, 903

Lane W. M., Cotton W. D., van Velzen S., Clarke T. E., Kassim N. E., Helmboldt J. F., Lazio T. J. W., Cohen A. S., 2014, MNRAS, 440, 327

Langston G. I., Heflin M. B., Conner S. R., Lehar J., Carilli C. L., Burke B. F., 1990, ApJS, 72, 621

Large M. I., Mills B. Y., Little A. G., Crawford D. F., Sutton J. M., 1981, MNRAS, 194, 693

Larionov M. G., 1991, Soobshcheniya Spetsial'noj Astrofizicheskoj Observatorii, 68, 14

Laurent-Muehleisen S. A., Kollgaard R. I., Ryan P. J., Feigelson E. D., Brinkmann W., Siebert J., 1997, A\&AS, 122

Magliocchetti M., Lutz D., et al., 2014, MNRAS, 442, 682

Malkin Z. M., 2016, Astronomy Reports, 60, 996

Malkin Z., Titov O., 2008, in Finkelstein A., Behrend D., eds, Measuring the Future, Proceedings of the Fifth IVS General Meeting. p. 183 (arXiv:0911.3216)

McMahon R. G., Omont A., Bergeron J., Kreysa E., Haslam C. G. T., 1994, MNRAS, 267, L9

Middelberg E., Deller A., et al., 2011, A\&A, 526, A74

Miley G., De Breuck C., 2008, A\&A Rev., 15, 67

Mingaliev M. G., Sotnikova Y. V., Torniainen I., Tornikoski M., Udovitskiy R. Y., 2012, A\&A, 544, A25

Mingaliev M. G., Sotnikova Y. V., Mufakharov T. V., Erkenov A. K., Udovitskiy R. Y., 2013, Astrophysical Bulletin, 68, 262

Mohan N., Rafferty D., 2015, PyBDSM: Python Blob Detection and Source Measurement, Astrophysics Source Code Library (ascl:1502.007)

Momjian E., Petric A. O., Carilli C. L., 2004, AJ, 127, 587

Momjian E., Carilli C. L., Petric A. O., 2005, AJ, 129, 1809

Momjian E., Carilli C. L., McGreer I. D., 2008, AJ, 136, 344

Mooley K. P., et al., 2016, ApJ, 818, 105

Morganti R., Fogasy J., Paragi Z., Oosterloo T., Orienti M., 2013, Science, 341,1082

Mortlock D. J., Warren S. J., et al., 2011, Nature, 474, 616

Munro R. E. B., 1972, Australian Journal of Physics Astrophysical Supplement, 22, 1

Murgia M., 2003, PASA, 20, 19

Murgia M., Fanti C., Fanti R., Gregorini L., Klein U., Mack K.H., Vigotti M., 2002, New A Rev., 46, 307

Murphy T., et al., 2010, MNRAS, 402, 2403

Myers S. T., et al., 2003, MNRAS, 341, 1

O'Dea C. P., 1998, PASP, 110, 493

Ochsenbein F., Bauer P., Marcout J., 2000, A\&AS, 143, 23

Ofek E. O., Frail D. A., 2011, ApJ, 737, 45 
Omont A., McMahon R. G., Cox P., Kreysa E., Bergeron J., Pajot F., Storrie-Lombardi L. J., 1996, A\&A, 315, 1

Omont A., Willott C. J., Beelen A., Bergeron J., Orellana G., Delorme P., 2013, A\&A, 552, A43

Orienti M., 2016, Astronomische Nachrichten, 337, 9

Orienti M., Dallacasa D., 2014, MNRAS, 438, 463

Orienti M., Dallacasa D., Stanghellini C., 2007, A\&A, 475, 813

Osmer P. S., 2004, Coevolution of Black Holes and Galaxies, p. 324

Owen F. N., Mufson S. L., 1977, AJ, 82, 776

Owen F. N., Porcas R. W., Mufson S. L., Moffett T. J., 1978, AJ, 83, 685

Paragi Z., Frey S., Gurvits L. I., Kellermann K. I., Schilizzi R. T., McMahon R. G., Hook I. M., Pauliny-Toth I. I. K., 1999, A\&A, 344, 51

Pariiskii I. N., Bursov N. N., Lipovka N. M., Soboleva N. S., Temirova A. V., Chepurnov A. V., 1992, A\&AS, 96, 583

Parijskij Y. N., Goss W. M., Kopylov A. I., Soboleva N. S., Temirova N. S., Verkhodanov O. V., Zhelenkova O. P., Naugolnaya M. N., 1996, Bulletin of the Special Astrophysics Observatory, 40,5

Parijskij Y. N., Kopylov A. I., Temirova A. V., Soboleva N. S., Zhelenkova O. P., Verkhodanov O. V., Goss W. M., Fatkhullin T. A., 2010, Astronomy Reports, 54, 675

Parijskij Y. N., et al., 2014, MNRAS, 439, 2314

Pâris I., et al., 2017, A\&A, 597, A79

Patnaik A. R., Browne I. W. A., Wilkinson P. N., Wrobel J. M., 1992, MNRAS, 254, 655

Pedani M., 2003, New Astron., 8, 805

Petric A. O., Carilli C. L., Bertoldi F., Fan X., Cox P., Strauss M. A., Omont A., Schneider D. P., 2003, AJ, 126, 15

Petrov L., Kovalev Y. Y., Fomalont E. B., Gordon D., 2006, AJ, 131, 1872

Petrov L., Kovalev Y. Y., Fomalont E. B., Gordon D., 2008, AJ, 136,580

Planck Collaboration et al., 2011, A\&A, 536, A7

Pushkarev A. B., Kovalev Y. Y., 2012, A\&A, 544, A34

Reich W., Fürst E., Reich P., Kothes R., Brinkmann W., Siebert J., 2000, A\&A, 363, 141

Rengelink R. B., Tang Y., de Bruyn A. G., Miley G. K., Bremer M. N., Röettgering H. J. A., Bremer M. A. R., 1997, A\&AS, 124,259

Richards J. L., et al., 2011, ApJS, 194, 29

Röettgering H. J. A., Lacy M., Miley G. K., Chambers K. C., Saunders R., 1994, A\&AS, 108

Romani R. W., 2006, AJ, 132, 1959

Romani R. W., Sowards-Emmerd D., Greenhill L., Michelson P., 2004, ApJ, 610, L9

Scaife A. M. M., Heald G. H., 2012, MNRAS, 423, L30

Singh V., Beelen A., et al., 2014, A\&A, 569, A52

Slee O. B., 1995, Australian Journal of Physics, 48, 143

Smolčić V., Ciliegi P., et al., 2014, MNRAS, 443, 2590

Snellen I. A. G., Schilizzi R. T., Miley G. K., de Bruyn A. G., Bremer M. N., Röttgering H. J. A., 2000, MNRAS, 319, 445

Spoelstra T. A. T., Patnaik A. R., Gopal-Krishna 1985, A\&A, 152,38

Sramek R. A., Weedman D. W., 1986, ApJ, 302, 640

Urry M., 1999, in Takalo L. O., Sillanpää A., eds, Astronomical Society of the Pacific Conference Series Vol. 159, BL Lac Phenomenon. p. 3 (arXiv:astro-ph/9812420)

Van Breugel W., De Breuck C., Stanford S. A., Stern D., Röttgering H., Miley G., 1999, ApJ, 518, L61

Van Weeren R. J., Williams et al., 2014, ApJ, 793, 82

Veres P., Frey S., Paragi Z., Gurvits L. I., 2010, A\&A, 521, A6

Verkhodanov O. V., Khabibullina M. L., 2010, Astronomy Letters, 36,7

Vollmer B., Davoust E., Dubois P., Genova F., Ochsenbein F., van Driel W., 2005, A\&A, 431, 1177
Waddington I., Windhorst R. A., Cohen S. H., Partridge R. B., Spinrad H., Stern D., 1999, ApJ, 526, L77

Waldram E. M., Yates J. A., Riley J. M., Warner P. J., 1996, MNRAS, 282, 779

Wang R., et al., 2011, ApJ, 739, L34

White R. L., Becker R. H., Helfand D. J., Gregg M. D., 1997, ApJ, 475, 479

Whitfield G. R., 1957, MNRAS, 117, 680

Wieringa M. H., Katgert P., 1992, A\&AS, 93, 399

Williams W. L., et al., 2016, MNRAS, 460, 2385

Worsley M. A., Fabian A. C., Pooley G. G., Chandler C. J., 2006, MNRAS, 368, 844

Wright A. E., Griffith M. R., Hunt A. J., Troup E., Burke B. F., Ekers R. D., 1996, ApJS, 103, 145

Xiang L., Stanghellini C., Dallacasa D., Haiyan Z., 2002, A\&A, 385,768

Yun M. S., Carilli C. L., Kawabe R., Tutui Y., Kohno K., Ohta K., 2000, ApJ, 528, 171

Zhang H.-Y., Liu X., Jin C.-J., Nan R.-D., 2001, Chinese J. Astron. Astrophys., 1, 129

\section{APPENDIX A: FLUX DENSITY REFERENCES}


Table A1. Flux density references

\begin{tabular}{|c|c|c|}
\hline Observation name & $\nu[\mathrm{MHz}]$ & Reference \\
\hline $4 \mathrm{C}$ & 178 & Gower et al. (1967) \\
\hline $7 \mathrm{C}$ & 151 & Waldram et al. (1996) \\
\hline $87 \mathrm{~GB}$ & 4850 & Gregory \& Condon (1991) \\
\hline AT20G & $4800 \& 8640 \& 19904$ & Murphy et al. (2010) \\
\hline ATATS & 1400 & Croft et al. (2010) \\
\hline B2.2 & 408 & Colla et al. (1972) \\
\hline B3 & 408 & Ficarra et al. (1985) \\
\hline BGP2002(V) & $2268 \& 8338$ & Beasley et al. (2002) \\
\hline CBR2001 & $1400 \& 250000$ & Carilli et al. (2001) \\
\hline CCW2015 & 325 & Coppejans et al. (2015) \\
\hline CFC2016(V) & $1658 \& 4990$ & Coppejans et al. (2016) \\
\hline CFG2014(V) & 1658 & Cao et al. (2014) \\
\hline CKK2002 & $1390 \& 42828$ & Carilli et al. (2002) \\
\hline CLASS & 8460 & Myers et al. (2003) \\
\hline CMM1999 & 1400 & Ciliegi et al. (1999) \\
\hline CNSS & 3000 & Mooley et al. (2016) \\
\hline CRATES & 8440 & Healey et al. (2007) \\
\hline CWH2007 & 233 & Carilli et al. (2007) \\
\hline f FIRST & 1400 & White et al. (1997) \\
\hline FFP2013 & 43000 & Frey et al. (2013) \\
\hline FFP2013(V) & 4850 & Frey et al. (2013) \\
\hline FGP2008(V) & $1600 \& 5000$ & Frey et al. (2008) \\
\hline FMP2003(V) & 1600 & Frey et al. (2003) \\
\hline FPF2015(V) & $1658 \& 4990$ & Frey et al. (2015) \\
\hline FPG2010(V) & $1658 \& 4990$ & Frey et al. (2010) \\
\hline FPG2011(V) & $1658 \& 4990$ & Frey et al. (2011) \\
\hline FPM2005 & $1400 \& 5000$ & Frey et al. (2005) \\
\hline FPM2005(V) & 5000 & Frey et al. (2005) \\
\hline GB6 & 4850 & Gregory et al. (1996) \\
\hline GCF2015(V) & 1658 & Gabányi et al. (2015) \\
\hline GMRT610 & 608 or 612 & This publication \\
\hline GMRT325 & 323 & This publication \\
\hline GMRT235 & 235 & This publication \\
\hline GMRT150 & 147 & This publication \\
\hline GOC1999 & 222068 & Guilloteau et al. (1999) \\
\hline HTT2007(V) & 4845 & Helmboldt et al. (2007) \\
\hline LHC1990 & 4830 & Langston et al. (1990) \\
\hline LKR1997 & 4885 & Laurent-Muehleisen et al. (1997) \\
\hline M1972 & 408 & Munro (1972) \\
\hline MCM2008 & 8400 & Momjian et al. (2008) \\
\hline MCM2008(V) & 1400 & Momjian et al. (2008) \\
\hline MCP2005 & 1400 & Momjian et al. (2005) \\
\hline MCP2005(V) & 1425 & Momjian et al. (2005) \\
\hline MPC2004(V) & 1425 & Momjian et al. (2004) \\
\hline MOB1994 & 240000 & McMahon et al. (1994) \\
\hline MOLONGLO & 408 & Large et al. (1981) \\
\hline NVSS & 1400 & Condon et al. (1998) \\
\hline OM1977 & 90000 & Owen \& Mufson (1977) \\
\hline OP1987 & $4585 \& 15064 \& 90000$ & Owen et al. (1978) \\
\hline OMC1996 & 239834 & Omont et al. (1996) \\
\hline OWB2013 & 250000 & Omont et al. (2013) \\
\hline PBW1992 & 8400 & Patnaik et al. (1992) \\
\hline PCB2003 & $1400 \& 5000$ & Petric et al. (2003) \\
\hline PFG1999(V) & 5000 & Paragi et al. (1999) \\
\hline PK2012(V) & $2300 \& 8600$ & Pushkarev \& Kovalev (2012) \\
\hline PKF2006(V) & $2309 \& 8646$ & Petrov et al. (2006) \\
\hline PKF2008(V) & $2309 \& 8646$ & Petrov et al. (2008) \\
\hline PKT2014(V) & $1658 \& 4994$ & Parijskij et al. (2014) \\
\hline
\end{tabular}


24 Rocco Coppejans et al.

Table A1 - continued

\begin{tabular}{ccc}
\hline Observation name & $\nu[\mathrm{MHz}]$ & Reference \\
\hline PMN & 4850 & Wright et al. (1996) \\
PTK2014(V) & $1658 \& 4994$ & Parijskij et al. (2014) \\
R2006 & $1440 \& 4880 \& 8440 \& 14950 \& 22490 \& 43330$ & Romani (2006) \\
RFR2000 & $2700 \& 5000 \& 10700$ & Reich et al. (2000) \\
RLM1994 & 1465 & Röettgering et al. (1994) \\
RMP2011 & 15000 & Richards et al. (2011) \\
RSG2004(V) & $15360 \& 43210$ & Romani et al. (2004) \\
S1995 & $80 \& 160$ & Slee (1995) \\
STRIPE82 & 1425 & Hodge et al. (2011) \\
TEXAS & 365 & Douglas et al. (1996) \\
TGSS & 148 & Intema et al. (2016) \\
VFP2010(V) & 15000 & Veres et al. (2010) \\
VLSSr & 74 & Lane et al. (2014) \\
WENSS & 325 & Rengelink et al. (1997) \\
WFP2006 & W & Worsley et al. (2006) \\
WWC2011 & 32000 & Wang et al. (2011) \\
WWR2016 & 149 & Williams et al. (2016) \\
WWT2014 & $62560 \& 22460 \& 43340$ & Van Weeren et al. (2014) \\
XSD2002(V) & 1660 & Xiang et al. (2002) \\
YCK2000 & $1400 \& 4900$ & Yun et al. (2000) \\
ZELENCHUK & 3900 & Larionov (1991) \\
ZLJ2001(V) & 1657 & Zhang et al. (2001) \\
\hline
\end{tabular}

